\title{
THE INFLUENCE OF DEUTERATION AND TURBULENT DIFFUSION ON THE OBSERVED D/H RATIO
}

\author{
T. A. Bell ${ }^{1}$, K. Willacy ${ }^{2}$, T. G. Phillips ${ }^{1}$, M. Allen ${ }^{2}$, and D. C. Lis ${ }^{1}$ \\ ${ }^{1}$ Department of Astronomy, California Institute of Technology, 1200 East California Boulevard, Pasadena, CA 91125, USA; tab@ caltech.edu \\ 2 Jet Propulsion Laboratory, California Institute of Technology, 4800 Oak Grove Drive, Pasadena, CA 91109, USA \\ Received 2010 May 16; accepted 2011 February 11; published 2011 March 22
}

\begin{abstract}
The influence of turbulent mixing on the chemistry of the interstellar medium (ISM) has so far received little attention. Previous studies of this effect have suggested that it might play an important role in mixing the various phases of the ISM. In this paper, we examine the potential effects of turbulent diffusion on the deuterium chemistry within molecular clouds. We find that such mixing acts to reduce the efficiency of deuteration in these clouds by increasing the ionization fraction and reducing freeze-out of heavy molecules. This leads to lower abundances for many deuterated species. We also examine the influence of turbulent mixing on the transition from atomic hydrogen to $\mathrm{H}_{2}$ and from atomic deuterium to HD near the cloud edge. We find that including turbulent diffusion in our models serves to push these transitions deeper into the cloud and helps maintain a higher atomic fraction throughout the cloud envelope. Based on these findings, we propose a new process to account for the significant scatter in the observed atomic $\mathrm{D} / \mathrm{H}$ ratio for galactic sightlines extending beyond the Local Bubble. Although several mechanisms have been put forward to explain this scatter, they are unable to fully account for the range in $\mathrm{D} / \mathrm{H}$ values. We suggest a scenario in which turbulent mixing of atomic and molecular gas at the edges of molecular clouds causes the observed atomic $\mathrm{D} / \mathrm{H}$ ratio to vary by a factor of $\sim 2$.
\end{abstract}

Key words: astrochemistry - diffusion - ISM: abundances - ISM: clouds - ISM: molecules - turbulence

Online-only material: color figures

\section{INTRODUCTION}

Since the first detection of a deuterium-bearing molecule, DCN, by Jefferts et al. (1973), it has become clear that the fraction of deuterium contained in molecules can be extremely high in the dense interstellar medium (ISM), with abundances of some deuterated species observed to be comparable to those of their hydrogenated counterparts. This is in stark contrast to the low atomic $\mathrm{D} / \mathrm{H}$ ratio measured by absorption studies in the diffuse gas, which has an average value of $(1.56 \pm 0.04) \times 10^{-5}$ (i.e., $15.6 \pm 0.4 \mathrm{ppm}$ ) in the local ISM (Wood et al. 2004).

The discovery of multiply deuterated molecules provided remarkable evidence to support the high molecular $\mathrm{D} / \mathrm{H}$ ratios observed. Based on the elemental $\mathrm{D} / \mathrm{H}$ ratio, such species would be expected to have abundances of order $10^{-10}$ or less, relative to their hydrogenated analogs. Yet doubly deuterated ammonia, $\mathrm{ND}_{2} \mathrm{H}$, was found to have an abundance ratio $\left[\mathrm{ND}_{2} \mathrm{H}\right] /\left[\mathrm{NH}_{3}\right]$ of $0.5 \%$ in the dark cloud L134N (Roueff et al. 2000) and 3\% in the prestellar core 16293E (Loinard et al. 2001). Similarly, $\left[\mathrm{D}_{2} \mathrm{CO}\right] /\left[\mathrm{H}_{2} \mathrm{CO}\right]$ ratios ranging from $1 \%$ to $40 \%$ have been observed in low-mass protostars and prestellar cores (Ceccarelli et al. 1998; Loinard et al. 2002; Bacmann et al. 2003). Subsequent detections of $\mathrm{CHD}_{2} \mathrm{OH}$ (Parise et al. 2002), $\mathrm{D}_{2} \mathrm{~S}$ (Vastel et al. 2003), and $\mathrm{D}_{2} \mathrm{H}^{+}$(Vastel et al. 2004) showed similarly high abundance ratios. Amazingly, molecules bearing three deuterons have also been detected, the first of which, triply deuterated ammonia, was observed to have an abundance ratio $\left[\mathrm{ND}_{3}\right] /\left[\mathrm{NH}_{3}\right] \sim 0.1 \%$ in the dark cloud Barnard 1 (Lis et al. 2002) and in the Class 0 protostar NGC 1333 IRAS 4A (van der Tak et al. 2002). Triply deuterated methanol, $\mathrm{CD}_{3} \mathrm{OH}$, has also been detected in the low-mass protostar IRAS 16293-2422 with an abundance ratio $\left[\mathrm{CD}_{3} \mathrm{OH}\right] /\left[\mathrm{CH}_{3} \mathrm{OH}\right] \sim 1 \%$ (Parise et al. 2004). Such extreme enhancement of the $D / H$ ratio within molecules points to the dramatic efficiency of deuterium frac- tionation in cold dense molecular clouds, a process that was first proposed by Solomon \& Woolf (1973).

At the low temperatures found in the cores of molecular clouds $(\sim 10 \mathrm{~K})$, the process of fractionation acts to minimize the zero-point vibrational energy of molecules by swapping constituent atoms for their heavier isotopes. Exchange reactions therefore lead to the preferential substitution of $\mathrm{H}$ by $\mathrm{D}$ in molecules. Most gas-phase chemistry proceeds via ion-molecule reactions, the most important ion of which is $\mathrm{H}_{3}^{+}$. Deuteration of this species through the reaction

$$
\mathrm{H}_{3}^{+}+\mathrm{HD} \rightleftharpoons \mathrm{H}_{2} \mathrm{D}^{+}+\mathrm{H}_{2}+232 \mathrm{~K}
$$

is also preferred at low temperatures (Watson 1976). Reactions with abundant gas-phase species, such as $\mathrm{CO}, \mathrm{O}$, and $\mathrm{N}_{2}$, transfer the deuteron to other molecules and limit the amount of $\mathrm{H}_{2} \mathrm{D}^{+}$ that can be maintained, e.g.,

$$
\mathrm{H}_{2} \mathrm{D}^{+}+\mathrm{CO} \rightarrow \mathrm{DCO}^{+}+\mathrm{H}_{2}
$$

In order for deuteration to proceed further and allow the formation of multiply deuterated species, $\mathrm{H}_{2} \mathrm{D}^{+}$must survive long enough for it to undergo subsequent deuteron exchange:

$$
\begin{aligned}
& \mathrm{H}_{2} \mathrm{D}^{+}+\mathrm{HD} \rightleftharpoons \mathrm{D}_{2} \mathrm{H}^{+}+\mathrm{H}_{2}+187 \mathrm{~K}, \\
& \mathrm{D}_{2} \mathrm{H}^{+}+\mathrm{HD} \rightleftharpoons \mathrm{D}_{3}^{+}+\mathrm{H}_{2}+234 \mathrm{~K} .
\end{aligned}
$$

This is possible when the atoms and molecules that destroy it are removed from the gas phase by freeze-out onto grain surfaces (Brown \& Millar 1989; Roueff et al. 2000; Roberts \& Millar 2000b). This occurs in the cold dense regions of the ISM and the resulting depletion of abundant species, such as $\mathrm{CO}$, has been observed in many sources (e.g., Willacy et al. 1998; Kramer et al. 1999; Caselli et al. 1999; Bergin et al. 2002; Bacmann et al. 2002; Tafalla et al. 2004). Direct evidence for 
the link between freeze-out and enhanced deuteration comes from the observed correlation between $\mathrm{CO}$ depletion and the $\left[\mathrm{D}_{2} \mathrm{CO}\right] /\left[\mathrm{H}_{2} \mathrm{CO}\right]$ ratio (Bacmann et al. 2003).

Grain-surface deuteration occurs by hydrogen abstraction and deuterium addition to mantle species on dust grains (Tielens 1983). This requires a reservoir of atomic deuterium to be maintained in the gas. This, again, is produced as a result of the $\mathrm{H}_{3}^{+} \rightarrow \mathrm{H}_{2} \mathrm{D}^{+} \rightarrow \mathrm{D}_{2} \mathrm{H}^{+} \rightarrow \mathrm{D}_{3}^{+}$series of reactions, with atomic deuterium a product of the dissociative recombination of these ions. For a more detailed discussion of deuterium chemistry, see, e.g., Roberts et al. (2003, 2004), Phillips \& Vastel (2003), and Phillips \& Lis (2006).

Studies of the atomic $\mathrm{D} / \mathrm{H}$ ratio in the local ISM provide an important constraint for models of galactic chemical evolution and insight into the processing of primordial gas up to the present era. Values for the galactic $\mathrm{D} / \mathrm{H}$ ratio are obtained by deriving column densities of D I and H I from observed Lyman series absorption lines in interstellar gas toward background stars. Since the first of these measurements were carried out, an unexpectedly large variation has emerged for sightlines extending beyond the Local Bubble, with values ranging from 5 to $22 \mathrm{ppm}$ (see, e.g., Linsky et al. 2006, for a recent discussion). This significant scatter is at odds with the more uniform values obtained for the primordial $\mathrm{D} / \mathrm{H}$ ratio, as probed by quasar absorption line systems and inferred from cosmic microwave background anisotropy data. These methods have yielded a fairly consistent value of $(\mathrm{D} / \mathrm{H})_{\text {prim }}=27.5_{-1.9}^{+2.4} \mathrm{ppm}$ (see, e.g., Cyburt et al. 2003).

Other than big bang nucleosynthesis, no formation mechanism for deuterium is known to exist, and explanations for the varying $\mathrm{D} / \mathrm{H}$ ratio therefore focus on ways to destroy or hide the deuterium. A corollary of this is that the highest observed values for the $\mathrm{D} / \mathrm{H}$ ratio must be closest to the true value. The process of astration - the conversion of deuterium to ${ }^{3} \mathrm{He},{ }^{4} \mathrm{He}$, and heavier elements via stellar nucleosynthesis-has long been proposed to explain the generally low values for the atomic $\mathrm{D} / \mathrm{H}$ ratio measured in the Galaxy, with differences in the degree of processing of the ISM being suggested as an explanation for the observed scatter. An alternative theory has been proposed by Draine $(2004,2006)$ in which deuterium atoms deplete onto dust grains in quiescent regions of the ISM and are subsequently returned to the gas phase if the grain mantles are destroyed. The observed correlation between metal depletion and decrease in the $\mathrm{D} / \mathrm{H}$ ratio has been used to support this theory (Prochaska et al. 2005; Linsky et al. 2006; Steigman et al. 2007), which predicts that regions that have experienced some degree of dust destruction should display both higher gas-phase abundances of refractory metals (titanium, iron, silicon, etc.) and higher $\mathrm{D} / \mathrm{H}$ ratios (owing to the removal of both metals and deuterium from the grains). There are, however, outliers in these trends that disrupt the correlation and cannot be explained by simple dust depletion models. One important process acts to increase the $\mathrm{D} / \mathrm{H}$ ratio, namely, the galactic infall of nearly pristine (i.e., unprocessed) material (see, e.g., Prodanović \& Fields 2008; Tosi 1996). If the infalling material is not fully mixed with the existing ISM, these inhomogeneities can also lead to variations in the observed $\mathrm{D} / \mathrm{H}$ ratio.

It is well established that molecular clouds are turbulent and that turbulence plays an important-perhaps even dominantrole in governing the dynamics and support of these clouds. What is less certain is the extent to which turbulent eddies can transport the gas and dust within a cloud, disrupting the abundance profiles predicted by static chemical models and redistributing the atomic and molecular species. The effects of turbulence on cloud chemistry were first considered by Phillips \& Huggins (1981). Subsequent work on this subject (e.g., Boland \& de Jong 1982; Federman \& Allen 1991; Xie et al. 1995; Willacy et al. 2002; Lesaffre et al. 2007) has demonstrated that turbulent mixing under conditions that are typical of molecular clouds can lead to "smearing out" of the abundance gradients, reducing the variation with depth as the gas is transported between the cloud envelope and its interior.

Turbulent transport in molecular clouds is a diffusive process (often called eddy diffusion; see, e.g., Hinze 1975) and the model employed in this work is derived from atmospheric chemical models in which the rate of turbulent transport for a given molecule depends on its abundance gradient. Such models describe the turbulent mixing between adjacent zones as a Fickian diffusion process with a diffusion coefficient $K$ derived from mixing length theory (Taylor 1915; Prandtl 1925), a phenomenological approach analogous to the concept of the mean free path in thermodynamics. For full details of this model, the reader is referred to the description contained in the original paper by Xie et al. (1995).

As already discussed, the process of deuterium fractionation is extremely efficient at altering the $\mathrm{D} / \mathrm{H}$ ratio of molecules in the cold, dense cores of molecular clouds. Indeed, Phillips $\&$ Lis (2006) suggest that this process may lead to a significant fraction of deuterium being locked in molecules in cloud centers. Meanwhile, at the edges of these clouds and in diffuse regions of the ISM, where the gas is warm and purely atomic, the observed atomic D/H ratio should reflect the "true" elemental value (neglecting, for the moment, the possibility that deuterium may be also depleted onto dust grains). Turbulent diffusion serves to mix these two phases of the ISM-the warm diffuse material at the edge of clouds and the cold dense material in their centers - and in so doing, may alter the atomic D/H ratio observed at the cloud edge, as well as that in the cloud center. If this is the case, then the observed atomic $\mathrm{D} / \mathrm{H}$ ratio along a particular line of sight will depend on a number of factors: (1) the degree of astration that the material along that sightline has been subjected to; (2) the amount of deuterium that has been depleted onto dust grains; (3) the efficiency of chemical fractionation in the gas along the sightline; (4) the degree of turbulent mixing of the different phases of the ISM; and (5) the proximity of the line of sight to a molecular cloud. In general, these mechanisms act to lower the value of the observed atomic $\mathrm{D} / \mathrm{H}$ ratio. Therefore, the highest observed value must be closest to the true elemental abundance ratio.

We have examined the effects of turbulent diffusion on deuterium chemistry within molecular clouds and the resulting change in the abundances of key deuterated species. We also propose a process by which the variation in the measured atomic $\mathrm{D} / \mathrm{H}$ ratio along diffuse lines of sight can be explained by turbulent mixing of the gas observed along these sightlines with denser material in neighboring regions. We describe the modifications made to the existing model for this work and the physical parameters of the cloud models considered in Section 2. The results of these models are presented in Section 3 and their implications for the observed $\mathrm{D} / \mathrm{H}$ ratio are discussed in Section 4. Finally, we summarize our findings in Section 5.

\section{THE MODEL}

The models used in this work are extensions of those described in Xie et al. (1995) and Willacy et al. (2002). The 
Table 1

Initial Abundances

\begin{tabular}{lccc}
\hline \hline Species & $n_{i} / n$ & Species & $n_{i} / n$ \\
\hline $\mathrm{H}$ & 1.00 & $\mathrm{He}$ & 0.14 \\
$\mathrm{D}$ & $1.60 \times 10^{-5}$ & $\mathrm{C}^{+}$ & $7.30 \times 10^{-5}$ \\
$\mathrm{~N}$ & $2.14 \times 10^{-5}$ & $\mathrm{O}$ & $1.76 \times 10^{-4}$ \\
$\mathrm{Mg}^{+}$ & $7.00 \times 10^{-9}$ & $\mathrm{Fe}^{+}$ & $3.00 \times 10^{-9}$ \\
\hline
\end{tabular}

Note. $n=n(\mathrm{H})+2 n\left(\mathrm{H}_{2}\right)$.

Table 2

Cloud Parameters

\begin{tabular}{lcccc}
\hline \hline Model & $\begin{array}{c}r_{0} \\
(\mathrm{pc})\end{array}$ & $\begin{array}{c}n_{0} \\
\left(\mathrm{~cm}^{-3}\right)\end{array}$ & $\begin{array}{c}T_{\text {out }} \\
(\mathrm{K})\end{array}$ & $\begin{array}{c}T_{\text {in }} \\
(\mathrm{K})\end{array}$ \\
\hline 1 & 1.11 & 888 & 10 & 10 \\
2 & 4.00 & 250 & 100 & 10 \\
\hline
\end{tabular}

reader is referred to those papers for a detailed description of the implementation of turbulent diffusion in the model.

The diffusive transport appears as an additional term in the continuity equation for each species, at each depth step,

$$
\frac{\partial n_{i}}{\partial t}=P_{i}-L_{i}-\frac{\partial \phi_{i}}{\partial r}
$$

where $n_{i}$ is the number density of species $i, P_{i}$ and $L_{i}$ are its production and loss terms, respectively, and $\phi_{i}$ is its net transport flux in the radial direction, given by

$$
\phi_{i}\left(\mathrm{~cm}^{-2} \mathrm{~s}^{-1}\right)=-K n \frac{\partial x_{i}}{\partial r},
$$

where $n$ is the total proton number density $\left[n=n(\mathrm{H})+2 n\left(\mathrm{H}_{2}\right)\right]$, $x_{i}=n_{i} / n$ is the fractional abundance of species $i$, and $K$ is the turbulent diffusion coefficient $\left(\mathrm{cm}^{2} \mathrm{~s}^{-1}\right)$. All quantities are depth and time dependent, with the exception of the diffusion coefficient, which we assume to be constant across the cloud.

The strength of turbulent mixing in the model is governed by the turbulent diffusion coefficient $K$. It is difficult to accurately determine values for $K$, since the nature of turbulence in molecular clouds is still unclear. However, it is possible to derive an order-of-magnitude estimate for $K$ by assuming $K \sim\left\langle V_{\mathrm{t}} L\right\rangle$, where $V_{\mathrm{t}}$ is the turbulent velocity and $L$ is the correlation length of the diffusive process (see Xie et al. 1995, for details). Based on estimates of the correlation length in molecular clouds $(0.1-0.5 \mathrm{pc})$ and typical turbulent velocities $\left(0.1-1 \mathrm{~km} \mathrm{~s}^{-1}\right)$, we consider values of $K$ between 0 (i.e., no diffusion) and $10^{23} \mathrm{~cm}^{2} \mathrm{~s}^{-1}$.

In this work, our aim is to explore the potential impact of turbulent mixing on deuterium fractionation, rather than to make quantitative predictions for the resulting changes in abundances or fractionation ratios. Precise values of $K$ are therefore not important and we focus instead on exploring the plausible range of values that might be expected.

The chemical network used in the model includes gas-phase and grain-surface reactions, freeze-out onto grain surfaces, and evaporation of mantle species. We adopt the gas-phase reaction rates of the RATE99 release of the UMIST database for astrochemistry (Le Teuff et al. 2000). The treatment of freezeout, grain-surface chemistry, and desorption mechanisms are described in Willacy (2007). We include the effects of thermal evaporation and cosmic-ray-induced desorption, and consider grain-surface reactions involving the addition of atoms and $\mathrm{CH}$,
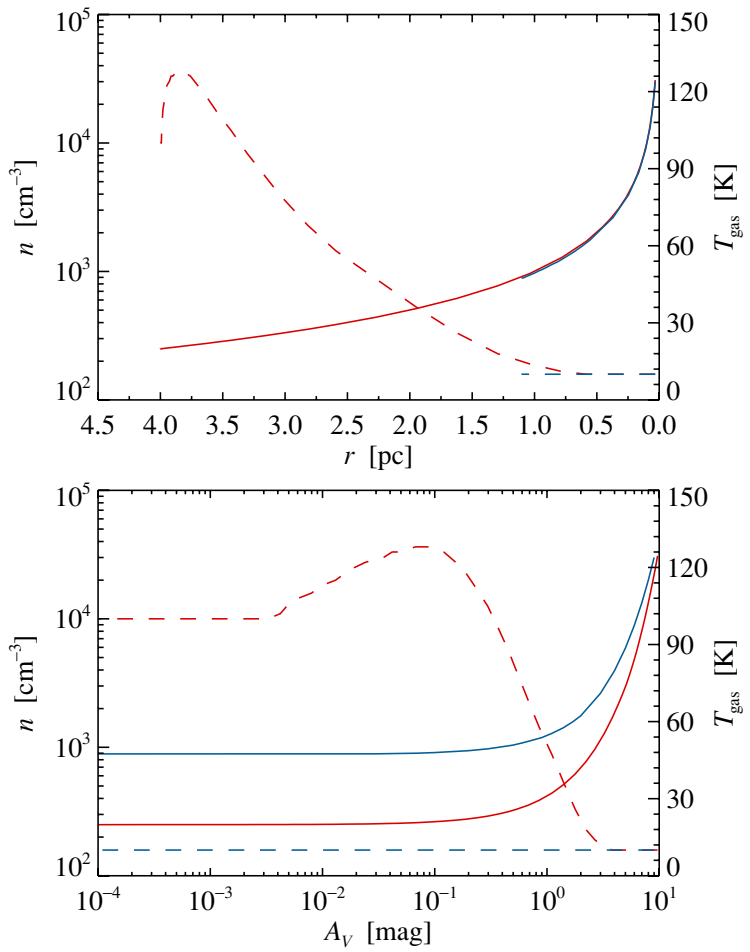

Figure 1. Cloud densities (solid lines) and gas temperatures (dashed lines) adopted in model 1 (blue) and model 2 (red). The top plot shows the radial dependence of these cloud properties and the bottom plot shows their variation as a function of visual extinction into the cloud.

(A color version of this figure is available in the online journal.)

$\mathrm{OH}$, and $\mathrm{NH}$ radicals to grain mantle species, as well as their deuterated analogs. The migration of $\mathrm{H}$ and $\mathrm{D}$ across grain surfaces is assumed to be governed by the relatively slow hopping between surface sites. Using slow rates with a rate equation model has been shown by Vasyunin et al. (2009) to give good agreement with more exact Monte Carlo models of grain-surface chemistry. The model also considers the effects of an external interstellar radiation field (ISRF) on the chemistry, including photodissociation and photoionization of gas-phase species and photoevaporation of mantle species from grain surfaces. Selfshielding by $\mathrm{H}_{2}$ increases the attenuation of dissociating farultraviolet (FUV) radiation, causing its photodissociation rate to drop rapidly with increasing depth into the cloud. We adopt the standard ISRF determined by Draine (1978) and the $\mathrm{H}_{2}$ selfshielding treatment of Lee et al. (1996). HD does not self-shield, but some of its lines do overlap with those of $\mathrm{H}_{2}$. This means that $\mathrm{H}_{2}$ can provide some shielding. Here we follow the approach of Barsuhn (1977) who estimated that the overlap of HD and $\mathrm{H}_{2}$ lines would reduce the HD photodissociation rate by $1 / 3$, assuming that the overlapped HD lines are totally shielded by the $\mathrm{H}_{2}$ lines.

The initial gas-phase elemental abundances used in all models are listed in Table 1. Initial abundances for all other species are assumed to be zero. We adopt the average elemental deuterium abundance determined for the Local Bubble $\left(1.6 \times 10^{-5}\right.$; Wood et al. 2004). Although this value is only valid locally, the true elemental deuterium abundance for the galactic disk is unknown (and likely to be higher). The effect of an increased deuterium abundance on the deuterium fractionation has been investigated by Roueff et al. (2007) who find that it leads to a corresponding linear increase in the deuteration of molecules. 

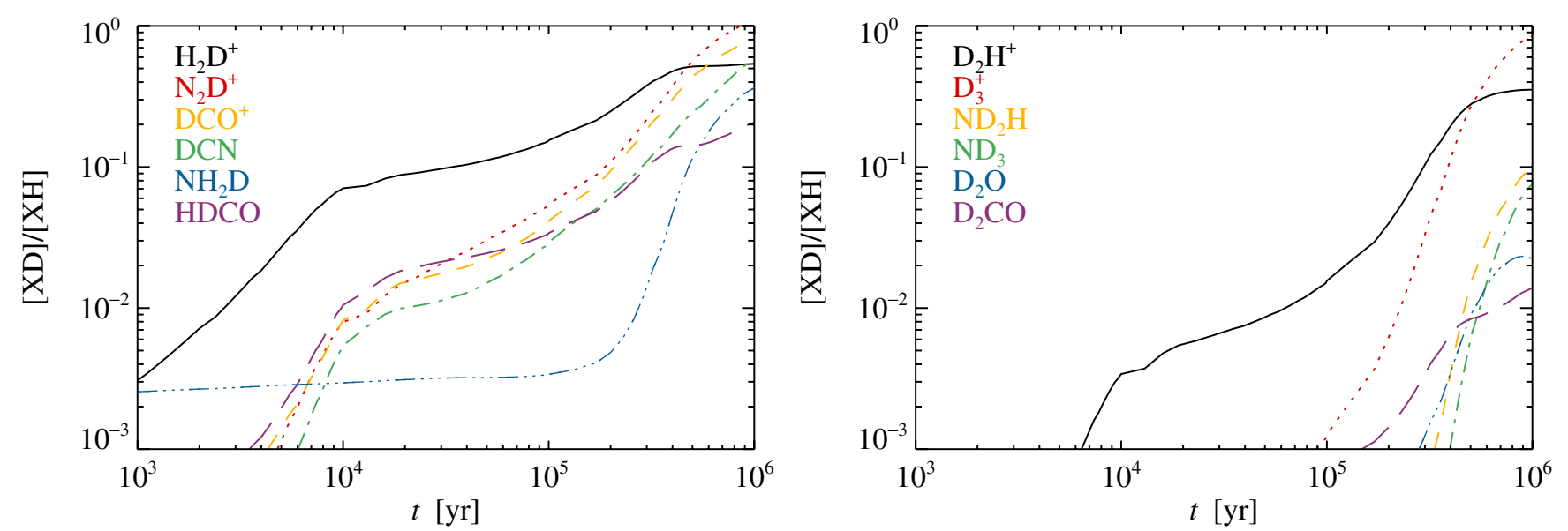

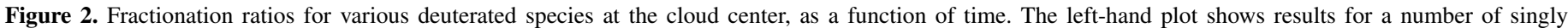

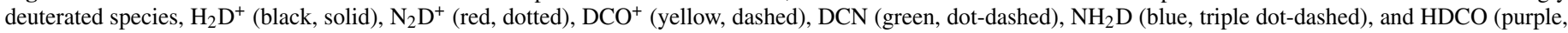

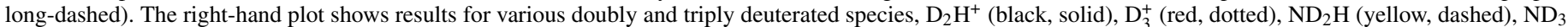
(green, dot-dashed), $\mathrm{D}_{2} \mathrm{O}$ (blue, triple dot-dashed), and $\mathrm{D}_{2} \mathrm{CO}$ (purple, long-dashed). Results for model 1 are shown.

(A color version of this figure is available in the online journal.)

\subsection{Changes to the Model}

The major modifications to the code necessary for this work were the introduction of deuterated species into the network of chemical reactions and the inclusion of freeze-out, grain surface chemistry, and desorption processes. The treatment of deuterium chemistry and these additional processes is described in detail in Willacy (2007).

\subsection{Cloud Properties}

In order to investigate the effects of turbulent diffusion under different interstellar environments, we have applied our code to two distinct sets of cloud parameters, which we list in Table 2. We assume a density profile of the form $n(r)=n_{0}\left(r_{0} / r\right)$ in both models. Since the code does not attempt to solve the thermal balance within the cloud, we impose fixed temperature profiles on the gas and dust. The models are distinct in the following ways. In model 1 , which is identical to that used previously by Xie et al. (1995) and Willacy et al. (2002), we adopt a cloud size of $r_{0}=1.11 \mathrm{pc}\left(3.41 \times 10^{18} \mathrm{~cm}\right)$, a number density at the cloud edge of $n_{0}=888 \mathrm{~cm}^{-3}$, and a constant gas and dust temperature of $10 \mathrm{~K}$ (i.e., $T_{\text {in }}=T_{\text {out }}=10 \mathrm{~K}$ ). Model 2 attempts to simulate a more extended cloud, with a larger, diffuse envelope extending to $r_{0}=4.0 \mathrm{pc}\left(1.23 \times 10^{19} \mathrm{~cm}\right)$ and a density at its edge of $n_{0}=250 \mathrm{~cm}^{-3}$. To account for the higher temperatures present in diffuse cloud envelopes, the temperature profile used in model 2 was determined using a separate code, designed to calculate the chemical and thermal structure of photondominated regions (PDRs) by treating the depth-dependent chemistry, heating, and cooling simultaneously (see Bell et al. 2006 for details). The same chemical network and incident radiation field were used in the PDR code and the density profile for model 2 was adopted. The resultant temperature profile initially rises sharply from $T_{\text {out }}=100 \mathrm{~K}$ at the cloud edge to $\sim 130 \mathrm{~K}$ at a radius of $3.8 \mathrm{pc}$, before gradually falling to $T_{\mathrm{in}}=10 \mathrm{~K}$ at the cloud center. The gas density and temperature profiles for models 1 and 2 are shown in Figure 1. In both models, the dust temperature is assumed to be constant at $10 \mathrm{~K}$ throughout the cloud. We note that, while this may only be truly valid for the dense central regions, the choice of dust temperature in the cloud envelope has less of an impact on the chemistry because freeze-out is less effective at the lower densities found there.

In all models, the chemical evolution of the cloud is computed for a period of 1 Myr. The results are discussed in the following section, where all abundances are quoted relative to the total proton number density, $n=n(\mathrm{H})+2 n\left(\mathrm{H}_{2}\right)$.

\section{RESULTS}

Before examining the effects of turbulent diffusion on the deuterium chemistry, it is important to compare the results of our static chemical model to previous theoretical work and to observations. Chemical models with increasingly sophisticated treatment of the deuterium fractionation and freeze-out have been constructed over the years (e.g., Roberts \& Millar 2000a, 2000b; Rodgers \& Charnley 2001; Roberts et al. 2003; Flower et al. 2004, 2006a, 2006b; Aikawa et al. 2005; Roueff et al. 2007). These are generally time dependent, but often limited to a single spatial point with conditions appropriate for the centers of dark clouds, characterized by low temperature $(T \sim 10 \mathrm{~K})$, high density $\left(n \geqslant 10^{4} \mathrm{~cm}^{-3}\right)$, and high visual extinction $\left(A_{V} \sim 10 \mathrm{mag}\right)$. Most of these models do not consider the more extended envelopes of molecular clouds, where temperatures are higher and densities lower, and the material is exposed to the ISRF (however, see Le Petit et al. 2002). For the purposes of comparison, we show in Figure 2 the fractionation ratios of a number of deuterated species at the center of our model cloud, as a function of time. In this paper, we use the term "fractionation ratio," or $[\mathrm{XD}] /[\mathrm{XH}]$, to mean the abundance ratio of a deuterated species with respect to its fully hydrogenated counterpart. It can be seen that fractionation ratios for many deuterated species approach values of $\sim 10 \%$ or higher at the cloud center after $1 \mathrm{Myr}$ of chemical evolution. Particularly impressive are $\mathrm{N}_{2} \mathrm{D}^{+}$, which reaches a higher abundance than $\mathrm{N}_{2} \mathrm{H}^{+}$, and $\mathrm{ND}_{3}$, whose abundance is comparable to $\mathrm{ND}_{2} \mathrm{H}$. In contrast, the fractionation ratios at the outer edge of the cloud are $\sim 0.1 \%$ or less for most deuterated species.

The majority of models described in the literature employ much higher number densities than are considered here (typically of order $10^{6} \mathrm{~cm}^{-3}$, whereas our central cloud density is $3 \times 10^{4} \mathrm{~cm}^{-3}$ ). This makes a direct comparison difficult. 
Table 3

Model Fractionation Ratios for Selected Species and Typical Observed Values

\begin{tabular}{lccc}
\hline \hline Species & $\begin{array}{c}\text { Model }^{\mathrm{a}} \\
N(\mathrm{XD}) / N(\mathrm{XH})\end{array}$ & $\begin{array}{c}\text { Observed } \\
{[\mathrm{XD}] /[\mathrm{XH}]}\end{array}$ & References \\
& $(\%)$ & $(\%)$ & \\
\hline $\mathrm{H}_{2} \mathrm{D}^{+}$ & 28 & $\ldots$ & $\ldots$ \\
$\mathrm{D}_{2} \mathrm{H}^{+}$ & 12 & $\ldots$ & $\ldots$ \\
$\mathrm{D}_{2} \mathrm{H}^{+} / \mathrm{H}_{2} \mathrm{D}^{+}$ & 42 & $50-100$ & $1^{\mathrm{b}}$ \\
$\mathrm{N}_{2} \mathrm{D}^{+}$ & 19 & $10-30$ & 2,3 \\
$\mathrm{DCO}^{+}$ & 15 & $4-18$ & 4,5 \\
$\mathrm{DCN}^{+}$ & 9 & $4-6$ & 2,6 \\
$\mathrm{DNC}$ & 4 & $2-9$ & 2,7 \\
$\mathrm{NH}_{2} \mathrm{D}$ & 10 & $10-30$ & $5,8,9$ \\
$\mathrm{ND}_{2} \mathrm{H}$ & 2 & $0.5-4$ & $9,10,11$ \\
$\mathrm{ND}_{3}$ & 0.9 & $0.02-0.55$ & $9,12,13$ \\
$\mathrm{HDO}$ & 8 & $0.2-3$ & 14 \\
$\mathrm{D}_{2} \mathrm{O}$ & 0.2 & $\ldots$ & $\ldots$ \\
$\mathrm{HDCO}$ & 6 & $3-7$ & 2,6 \\
$\mathrm{D}_{2} \mathrm{CO}$ & 0.2 & $1-10$ & 15,16 \\
\hline
\end{tabular}

Notes.

a Model fractionation ratios are determined from total column densities integrated over all radial points in model 1 at $1 \mathrm{Myr}$.

${ }^{b}$ Observed fractionation ratios for $\mathrm{H}_{2} \mathrm{D}^{+}$and $\mathrm{D}_{2} \mathrm{H}^{+}$do not exist in the literature, but the $\left[\mathrm{D}_{2} \mathrm{H}^{+}\right] /\left[\mathrm{H}_{2} \mathrm{D}^{+}\right]$ratio has been measured in the prestellar core $16293 \mathrm{E}$ by Vastel et al. (2004).

References. (1) Vastel et al. 2004; (2) Turner 2001; (3) Gerin et al. 2001; (4) Williams et al. 1998; (5) Tiné et al. 2000; (6) Roberts et al. 2002; (7) Hirota et al. 2003; (8) Hatchell 2003; (9) Roueff et al. 2005; (10) Roueff et al. 2000; (11) Loinard et al. 2001; (12) Lis et al. 2002; (13) van der Tak et al. 2002; (14) Parise et al. 2005; (15) Bacmann et al. 2003; (16) Roberts \& Millar 2007.

Roberts et al. (2003) performed detailed chemical modeling that included multiply deuterated species and freeze-out of gasphase species onto grain mantles. They considered models with $T=10 \mathrm{~K}$ and $n\left(\mathrm{H}_{2}\right)=10^{4} \mathrm{~cm}^{-3}$, conditions very similar to those in the center of our model cloud. In order to compare our chemical model to theirs, we ran a single depth-step model with the same physical conditions and initial abundances as used in their paper. Comparing the fractionation ratios listed in their Table 1 with the values from our single-point model at $t=$ $1 \mathrm{Myr}$, the two models agree within a factor of two for most deuterated species, the only exception being $\mathrm{ND}_{3}$, which is an order of magnitude higher in our model. This disagreement is probably due to differences in the choice of branching ratios in the chemical networks. A detailed analysis of the differences between our model and those of others is beyond the scope of this paper, but despite these minor differences, we consider our model to be in good agreement with the results of Roberts et al. (2003).

When comparing our results to observational data, it is more appropriate to consider fractionation ratios based on total column densities through the model cloud, since observed fractionation ratios are determined in the same way. We therefore list in Table 3 the fractionation ratios for various species in the model determined from total column densities, $N(\mathrm{XD}) / N(\mathrm{XH})$, alongside the typical range of observed values quoted in the literature. The general agreement is very good, with model fractionation ratios for most species falling within, or very close to, the observed range of values. The notable exceptions are HDO, the bulk of which is expected to be in the form of ice, and $\mathrm{D}_{2} \mathrm{CO}$, which is typically observed in warmer sources or regions of higher density (Bacmann et al. 2003). The model values generally fall at the lower end of the observed ranges, which is to be expected, since the majority of the observed sources have much higher central densities.

Our intention in this paper is to examine the general effects of introducing turbulent diffusion into models of deuterium chemistry in the ISM, focusing on overall trends, rather than making quantitative predictions for specific sources. With this in mind, we regard the results of our deuterium model to be in good agreement with previous models and observations.

\subsection{General Trends}

We find that turbulent mixing can often lead to unintuitive effects on the deuterium chemistry, reducing the level of deuteration in some species, while enhancing that of others at certain points within the cloud. This is due to the complex interplay between mixing and chemical processes, redistributing important feed molecules that govern the deuterium chemistry, while disrupting the freeze-out of abundant species such as CO that can hinder deuteration of gas-phase species.

To illustrate the predicted effects of turbulent diffusion on the deuterium chemistry, we show in Figure 3 the fractionation ratios of a number of important deuterated molecules (at $t=1 \mathrm{Myr}$ ) as a function of visual extinction into the cloud for a set of models with diffusion coefficients of $K=0$ (i.e., no diffusion) to $K=10^{23} \mathrm{~cm}^{2} \mathrm{~s}^{-1}$ (an upper limit to the possible range of values). It is immediately obvious that increasing levels of turbulent diffusion generally act to reduce the abundances of deuterated molecules. In the cloud center $\left(A_{V}>1 \mathrm{mag}\right)$, all deuterated species suffer under the influence of turbulent diffusion, with fractionation ratios dropping by up to several orders of magnitude. However, the effect is not uniform throughout the cloud, and in the envelope, the deuteration of some species actually increases when turbulent mixing is active. In the following two sections, we consider the effects of turbulent diffusion on the cloud center and envelope separately.

\subsection{Cloud Core}

Turbulent diffusion limits the abundance of deuterated species in the cloud center by disrupting two of the key features that are important for effective deuteration.

1. The ionization fraction (electron abundance) is enhanced.

2. Depletion of gas-phase species onto grains is reduced.

Together, these lead to significantly lower abundances of $\mathrm{H}_{2} \mathrm{D}^{+}, \mathrm{D}_{2} \mathrm{H}^{+}$, and $\mathrm{D}_{3}^{+}$compared to chemical models that do not include turbulent mixing, in turn resulting in lower abundances of the deuterated molecules that are formed from these ions.

Figure 4 shows the fractional abundance of electrons as a function of visual extinction in both models, for values of $K$ from 0 (no diffusion) to $10^{23} \mathrm{~cm}^{2} \mathrm{~s}^{-1}$ (strong diffusion). The electron abundance in the cloud center rises by a factor of four when $K=10^{23}$, compared to models in which diffusion is absent. This is due to the influx of ions and electrons from the cloud surface, where species such as carbon are rapidly photoionized by the unattenuated flux of FUV photons.

Turbulent diffusion also acts to reduce the level of freeze-out in the cold dense cloud core. This is caused by movement of the grains to regions of lower extinction and density, where their mantles are released back into the gas, either by evaporation or photodesorption. As an example, gas-phase $\mathrm{CO}$ in the cloud center is over an order of magnitude more abundant in the $K=10^{23} \mathrm{~cm}^{2} \mathrm{~s}^{-1}$ models than in the models with no diffusion, while the grain mantle abundance of $\mathrm{CO}$ shows a corresponding drop (see Figure 5). Atomic oxygen, which rapidly destroys $\mathrm{H}_{3}^{+}$, 

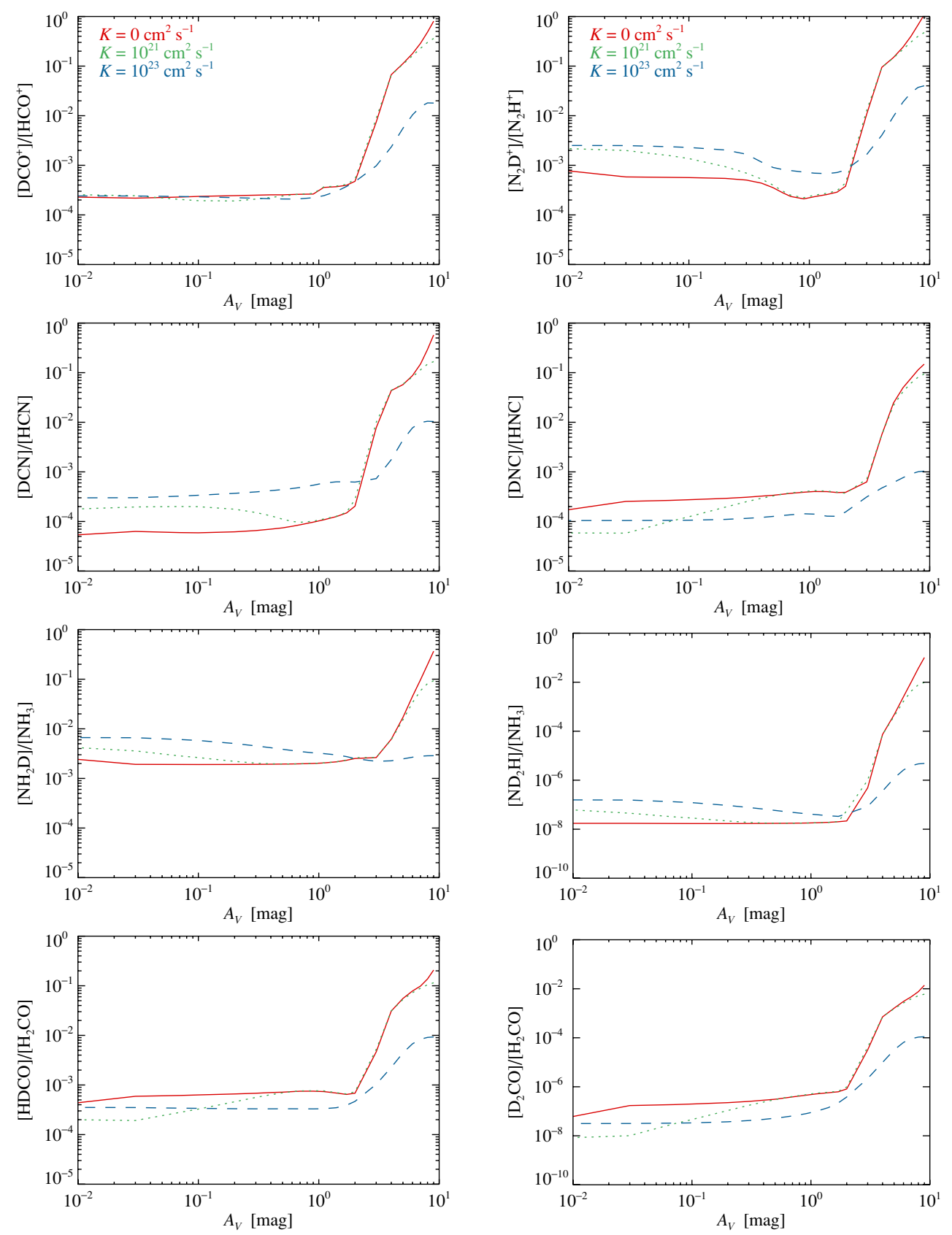

Figure 3. Fractionation ratios for $\mathrm{DCO}^{+}, \mathrm{N}_{2} \mathrm{D}^{+}, \mathrm{DCN}, \mathrm{DNC}, \mathrm{NH}_{2} \mathrm{D}, \mathrm{ND}_{2} \mathrm{H}, \mathrm{HDCO}$, and $\mathrm{D}_{2} \mathrm{CO}$ (from top left to bottom right, respectively) for diffusion coefficients of $K=0,10^{21}$, and $10^{23} \mathrm{~cm}^{2} \mathrm{~s}^{-1}$ (red solid, green dotted, and blue dashed lines, respectively). All plots show results for model 1 at $t=1 \mathrm{Myr}$.

(A color version of this figure is available in the online journal.)

is 700 times more abundant in the $K=10^{23}$ model because its freeze-out onto grains is reduced.

The combination of increased electron abundance and decreased freeze-out in the presence of turbulent diffusion leads to significantly lower abundances of $\mathrm{H}_{3}^{+}$and its deuterated analogs, compared to models with no diffusion. This in turn leads to lower abundances of the deuterated molecules that are formed from these ions. These trends are illustrated in Figure 6, which shows the abundances of $\mathrm{H}_{3}^{+}, \mathrm{H}_{2} \mathrm{D}^{+}$, and $\mathrm{D}_{2} \mathrm{H}^{+}$dropping as the level of turbulent diffusion rises. Comparing models with $K=0$ and $K=10^{23} \mathrm{~cm}^{2} \mathrm{~s}^{-1}$, the abundances of $\mathrm{H}_{3}^{+}, \mathrm{H}_{2} \mathrm{D}^{+}$, and $\mathrm{D}_{2} \mathrm{H}^{+}$at the cloud center drop by factors of approximately 10, 30, and 200 , respectively, when turbulent diffusion is active (for model 1 parameters; the results for model 2 show a similar trend).
Dissociative recombination of $\mathrm{H}_{2} \mathrm{D}^{+}$and its multiply deuterated isotopologues is also the primary source of $\mathrm{D}$ atoms in the cloud center. As their abundances are lowered under the presence of turbulent diffusion, so too is the abundance of atomic deuterium — by a factor of $\sim 10^{4}$ when $K=10^{23} \mathrm{~cm}^{2} \mathrm{~s}^{-1}$. The lower abundance of $\mathrm{D}$ then inhibits the deuteration of molecules on grain surfaces, which proceeds via deuteron addition to mantle species. Since the high levels of deuteration observed toward low-luminosity protostars are often attributed to evaporation of mantle species following active grain surface chemistry (e.g., Loinard et al. 2001; Roberts et al. 2003), the role of turbulent diffusion in limiting grain surface deuteration may have implications for the degree of fractionation seen in the gas phase at later stages of protostellar evolution. 

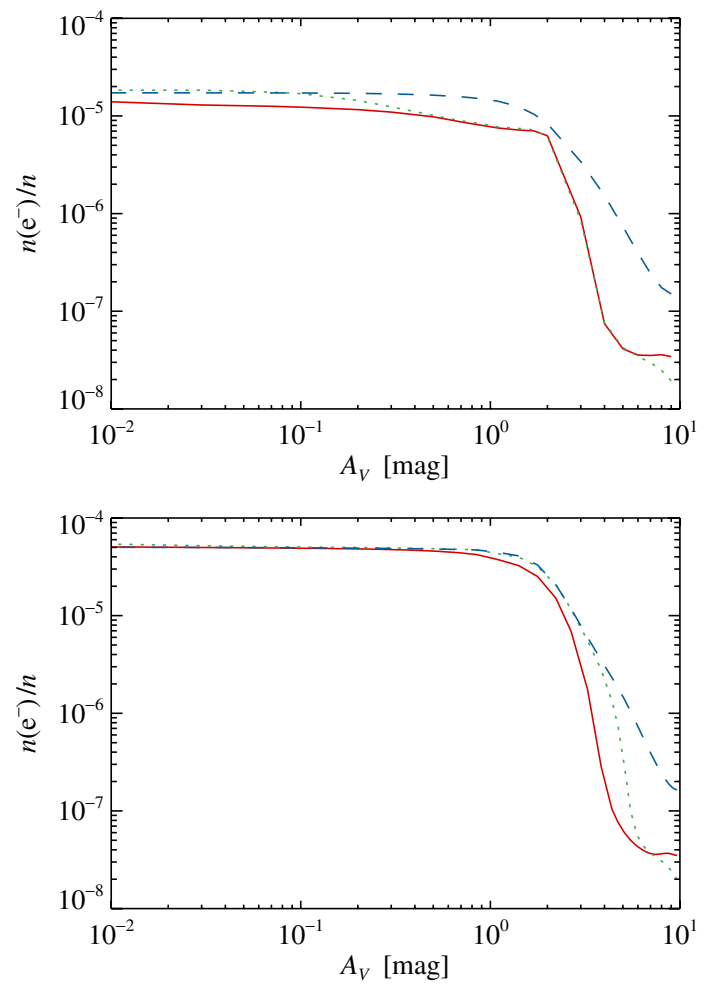

Figure 4. Electron fractional abundances for diffusion coefficients of $K=0$, $10^{21}$, and $10^{23} \mathrm{~cm}^{2} \mathrm{~s}^{-1}$ (red solid, green dotted, and blue dashed lines, respectively). The results for model 1 are shown in the top plot and those for model 2 in the bottom, both at $t=1 \mathrm{Myr}$.

(A color version of this figure is available in the online journal.)
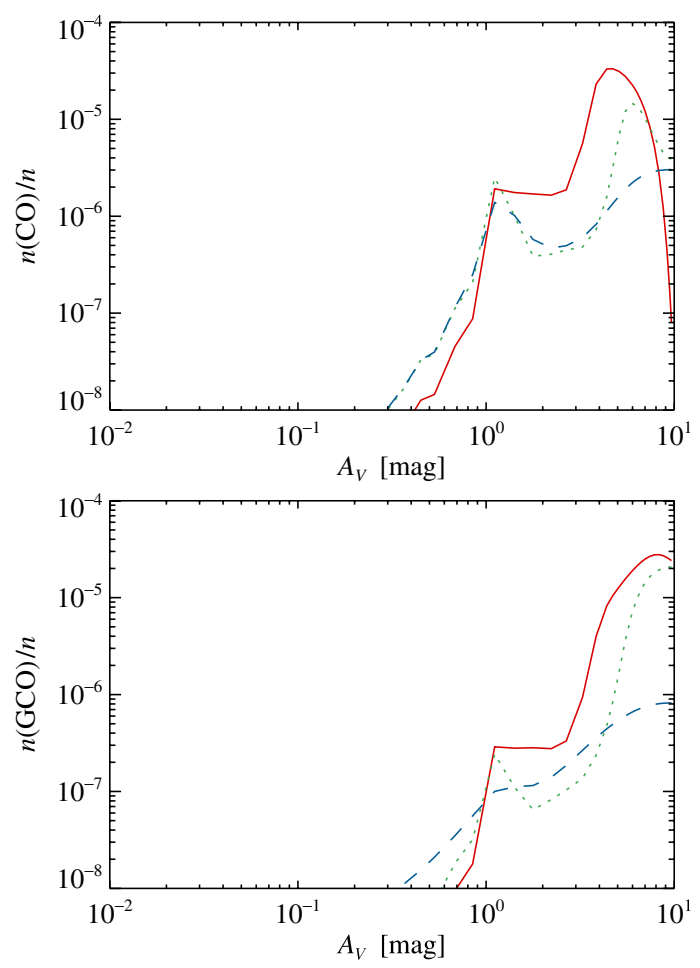

Figure 5. Fractional abundance of $\mathrm{CO}$, both in the gas phase and on grain surfaces (referred to as GCO), for diffusion coefficients of $K=0,10^{21}$, and $10^{23} \mathrm{~cm}^{2} \mathrm{~s}^{-1}$ (red solid, green dotted, and blue dashed lines, respectively). The results shown are for model 2 at $t=1 \mathrm{Myr}$.

(A color version of this figure is available in the online journal.)
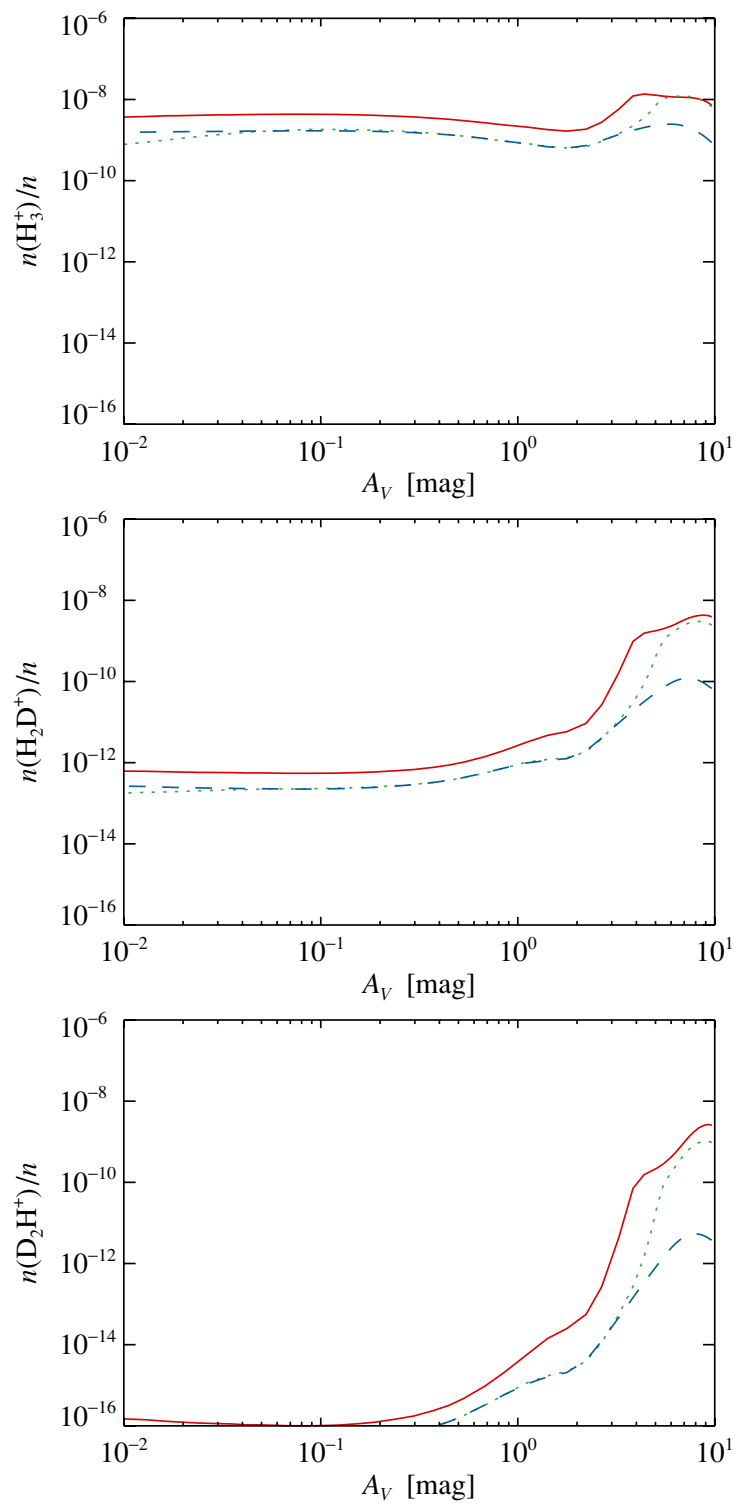

Figure 6. Fractional abundances of $\mathrm{H}_{3}^{+}, \mathrm{H}_{2} \mathrm{D}^{+}$, and $\mathrm{D}_{2} \mathrm{H}^{+}$for diffusion coefficients of $K=0,10^{21}$, and $10^{23} \mathrm{~cm}^{2} \mathrm{~s}^{-1}$ (red solid, green dotted, and blue dashed lines, respectively). The results shown are for model 2 at $t=1 \mathrm{Myr}$.

(A color version of this figure is available in the online journal.)

\subsection{Cloud Envelope}

At the cloud edge, diffusion affects the typical cloud chemistry by supplying fresh molecular gas to be photodissociated/ photoionized by the incident FUV photons, the products of which are then carried into the cloud interior. This increases the depth at which the gas becomes molecular, shifting the locations of the $\mathrm{H} / \mathrm{H}_{2}$ and $\mathrm{C}^{+} / \mathrm{C} / \mathrm{CO}$ transitions that are characteristic features in models of cloud edges. However, the influence of turbulent diffusion is not uniform on all species. An interesting result of this is that the location of the $\mathrm{H} / \mathrm{H}_{2}$ transition can occur deeper into the cloud than that of $\mathrm{D} / \mathrm{HD}$, despite the latter's weaker shielding against photodissociation. The reason for this lies in the importance of the abundance gradient in determining the rate of diffusion. Since the elemental abundance of hydrogen is orders of magnitude above that of deuterium, the abundance gradients of $\mathrm{H}$ and $\mathrm{H}_{2}$ at the cloud edge, where the abundance of $\mathrm{H}_{2}$ drops rapidly from 0.5 to $\sim 10^{-3}$, are much 

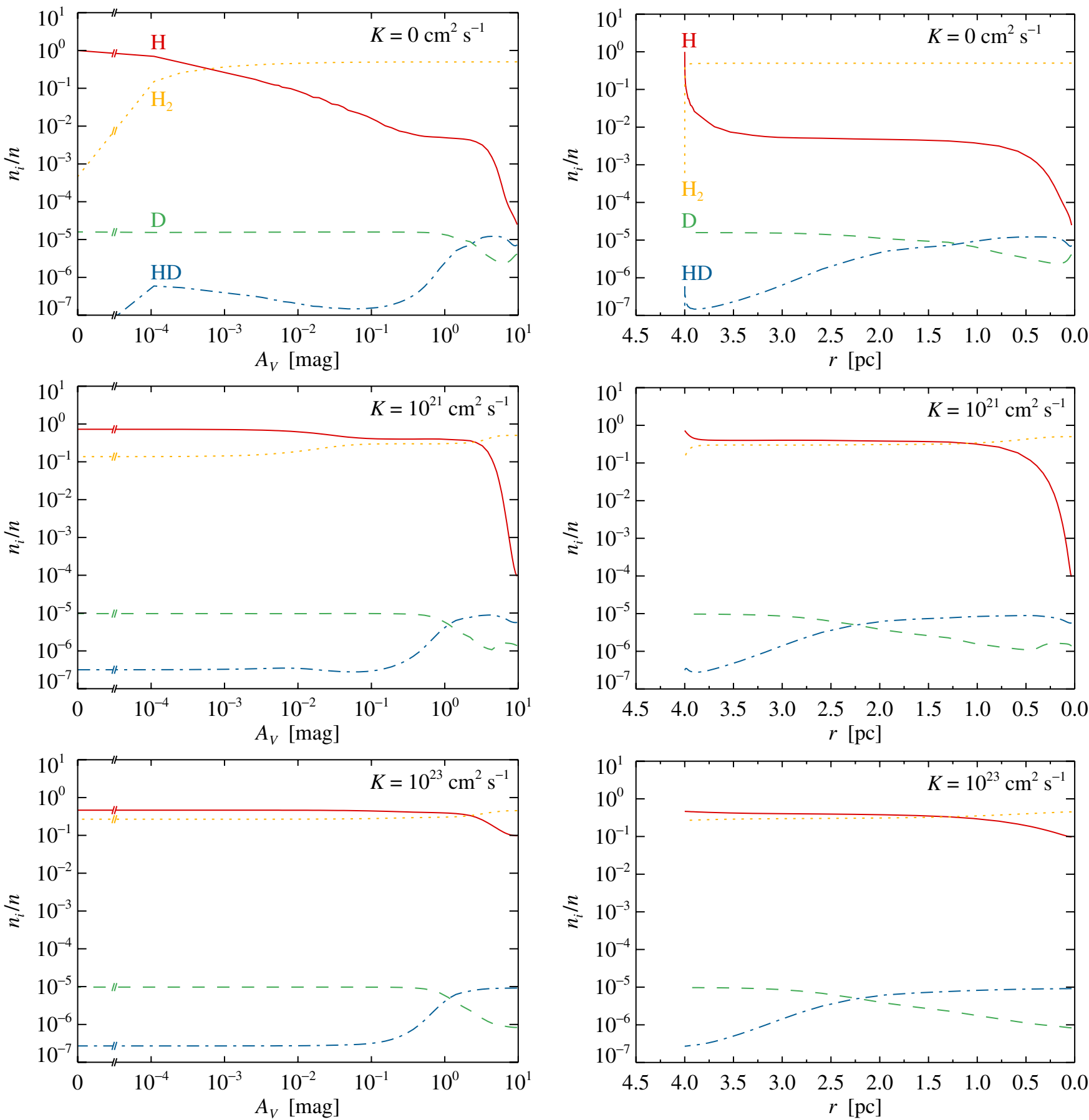

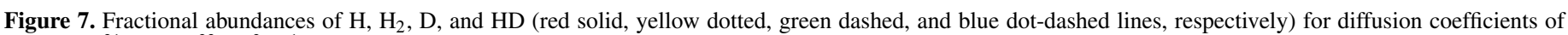
$K=0,10^{21}$, and $10^{23} \mathrm{~cm}^{2} \mathrm{~s}^{-1}$, from top to bottom. The results shown are for model 2 at $t=1 \mathrm{Myr}$.

(A color version of this figure is available in the online journal.)

steeper than those of D and HD. This is illustrated in Figure 7, where the abundances of $\mathrm{H}, \mathrm{H}_{2}, \mathrm{D}$, and $\mathrm{HD}$ are shown as a function of visual extinction into the cloud for models with diffusion coefficients of $K=0$ to $10^{23} \mathrm{~cm}^{2} \mathrm{~s}^{-1}$. The inward flux of atomic hydrogen from the cloud surface, where it is produced by photodissociation of (primarily) $\mathrm{H}_{2}$, is over four orders of magnitude greater than the inward flux of atomic deuterium. This has the effect of "smearing out" the otherwise sharp $\mathrm{H} / \mathrm{H}_{2}$ transition to such an extent that the depth at which $\mathrm{H}$ and $\mathrm{H}_{2}$ have equal abundances increases from $A_{V} \sim 7 \times 10^{-4}(K=0)$ to $A_{V} \sim 2\left(K=10^{23}\right)$. In contrast, the equivalent position for
D and HD moves only slightly, and in the opposite direction, from $A_{V} \sim 2$ to $A_{V} \sim 1 \mathrm{mag}$.

Under typical interstellar conditions, molecular hydrogen is believed to form exclusively on grain surfaces. HD, however, is not only formed on grains, but can also form efficiently in the gas phase by the reaction

$$
\mathrm{D}^{+}+\mathrm{H}_{2} \rightarrow \mathrm{H}^{+}+\mathrm{HD} \text {. }
$$

Indeed, in the warm diffuse cloud envelope, where the $\mathrm{D}^{+}$ abundance is high due to charge exchange between $\mathrm{D}$ and $\mathrm{H}^{+}$, and $\mathrm{H}_{2}$ begins to become abundant, this gas-phase reaction 


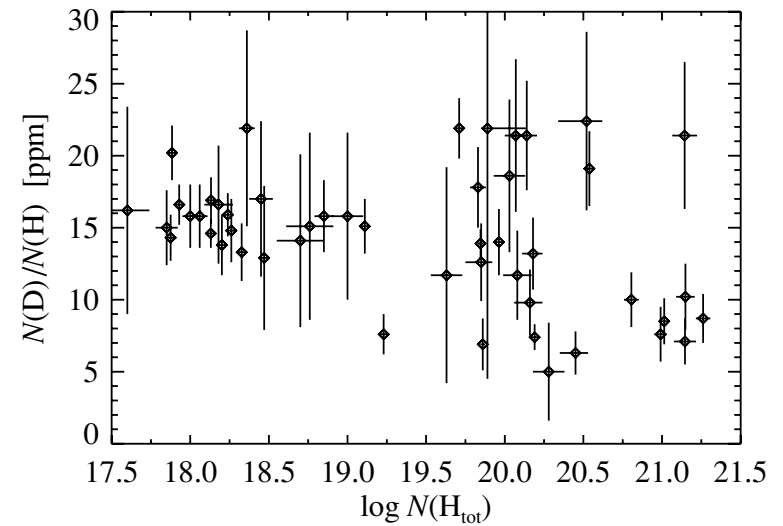

Figure 8. Observed galactic $[\mathrm{D}] /[\mathrm{H}]$ ratios as a function of line-of-sight total hydrogen column density $\left[N\left(\mathrm{H}_{\mathrm{tot}}\right)=N(\mathrm{HI})+2 N\left(\mathrm{H}_{2}\right)\right]$. See Table 4 for more details and references.

becomes the dominant formation route for HD. The jump in the HD abundance near the cloud edge $\left(A_{V} \sim 10^{-4} \mathrm{mag}\right.$, $r \sim 4 \mathrm{pc}$ ), seen in the top two plots of Figure 7 , is due to the combination of high abundances of both $\mathrm{D}^{+}$(not shown) and $\mathrm{H}_{2}$ at this point; nearer to the cloud surface, $\mathrm{H}_{2}$ is less abundant due to photodissociation, and further into the cloud, $\mathrm{D}^{+}$drops in abundance, due to the declining abundance of $\mathrm{H}$ and thus $\mathrm{H}^{+}$, from which $\mathrm{D}^{+}$forms.

With the introduction of turbulent diffusion, the abundance gradient of $\mathrm{D}^{+}$is smoothed out, such that its abundance remains more constant across the cloud envelope and is a factor of $\sim 3$ higher at $A_{V}=1 \mathrm{mag}$ when $K=10^{23}$. This, in turn, leads to increased gas-phase formation of HD at this cloud depth, resulting in a higher HD abundance and thus explaining the outward shift in the location of the D/HD transition. This change is relatively insensitive to the strength of turbulent diffusion; models with $K=10^{21}$ and $K=10^{23} \mathrm{~cm}^{2} \mathrm{~s}^{-1}$ produce nearly identical changes in the $\mathrm{D}^{+}$and HD abundances at this cloud depth.

While the diffusive process acts to redistribute atomic species throughout the cloud, it also brings molecular material from the interior out to the envelope of the cloud. This allows certain deuterated species to attain higher abundances within the region of low extinction than can be produced by static cloud models, where the effects of photodissociation, lower density, and higher temperature inhibit the formation of such species. A notable example of this is $\mathrm{N}_{2} \mathrm{D}^{+}$, which shows an abundance enhancement of almost an order of magnitude when $K=10^{23} \mathrm{~cm}^{2} \mathrm{~s}^{-1}$, compared to the static cloud model (at $\left.A_{V} \sim 10^{-2} \mathrm{mag}\right)$.

We note that observations of $\mathrm{H}_{3}^{+}$in diffuse clouds (e.g., McCall et al. 2003; Indriolo et al. 2007) suggest that large low-energy cosmic-ray ionization rates are needed to explain the observed high abundances. These rates are up to an order of magnitude greater than the standard rate considered in our model. Although it is beyond the scope of this paper to examine the possible influence of varying model parameters such as the cosmic-ray ionization rate on the deuterium chemistry, it is worth mentioning that an order of magnitude increase in the rate would lead to higher $\mathrm{H}_{3}^{+}$and electron abundances in the model, which would likely reduce the level of deuteration that could be achieved. Since turbulent diffusion is effective at redistributing species between the cloud core and envelope, this would likely lead to an overall reduction in the level of deuteration throughout the cloud.

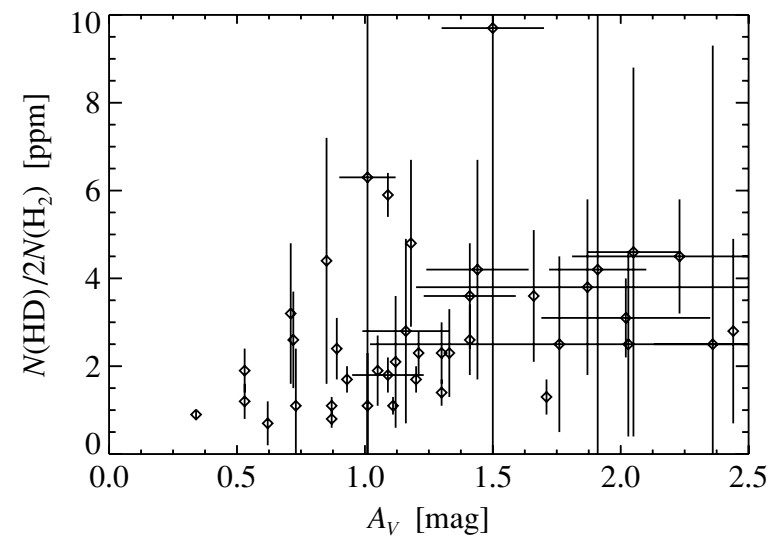

Figure 9. Observed galactic $[\mathrm{HD}] / 2\left[\mathrm{H}_{2}\right]$ ratios as a function of line-of-sight visual extinction $\left[A_{V}=R_{V} \cdot E(B-V)\right]$. See Table 5 for more details and references.

\section{IMPLICATIONS FOR THE OBSERVED D/H RATIO}

The galactic atomic $\mathrm{D} / \mathrm{H}$ ratio has been studied extensively by observing absorption lines of $\mathrm{D}$ I and $\mathrm{H}_{\mathrm{I}}$ toward background stars in the local ISM. In the past, the lines of sight were restricted to nearby stars $(d \lesssim 200 \mathrm{pc})$ by the sensitivity limits of the Copernicus and Hubble Space Telescope instruments, but with the launch of the Far-Ultraviolet Spectroscopic Explorer (FUSE) satellite, absorption studies along sightlines to stars several kpc away became possible. Figure 8 and Table 4 show a compilation of the observed galactic $\mathrm{D} / \mathrm{H}$ ratios, taken primarily from the compilation of Linsky et al. (2006), but with more recent measurements added. The scatter in observed values is significant, with some measurements up to a factor of two above or below the mean value.

More recently, the molecular $\mathrm{D} / \mathrm{H}$ ratio, defined as [HD] $/ 2\left[\mathrm{H}_{2}\right]$, has begun to be examined in more detail, since FUSE allows sightlines containing significant fractions of molecular material to be probed. Figure 9 and Table 5 show a compilation of the observed galactic $[\mathrm{HD}] / 2\left[\mathrm{H}_{2}\right]$ ratios, taken primarily from Snow et al. (2008), but updated with more recent data.

Let us now assume that our model cloud intersects a line of sight toward a background star and that we measure the column densities of $\mathrm{HI}_{\mathrm{I}}$ and $\mathrm{DI}_{\mathrm{I}}$ seen in absorption through the cloud. Given the abundance profiles of $\mathrm{H}, \mathrm{H}_{2}, \mathrm{D}$, and $\mathrm{HD}$ discussed in the previous section, what atomic $\mathrm{D} / \mathrm{H}$ ratios might we expect at different points within the cloud? If the cloud consisted purely of atomic gas, measurements along any line of sight through it would yield the true elemental $\mathrm{D} / \mathrm{H}$ ratio (i.e., that specified as an input to the model). However, if we consider a model cloud containing cold, dense material, much of the hydrogen and deuterium will be contained in molecules, either in the gas phase or on grain mantles. In this case, the measured atomic $\mathrm{D} / \mathrm{H}$ ratio will no longer necessarily reflect the true elemental $\mathrm{D} / \mathrm{H}$ ratio. Instead, it will reflect the remaining amount of hydrogen and deuterium in atomic form along a particular sightline, and will no longer "see" the deuterium and hydrogen contained in molecules. At this point, the measured $\mathrm{D} / \mathrm{H}$ ratio becomes dependent upon the various chemical reactions that form and remove atomic deuterium and hydrogen. Due to the mass difference between these isotopologues, the formation and destruction rates for $\mathrm{H}$ - and $\mathrm{D}$-bearing species will differ at low temperatures as a result of fractionation, and the fraction of each that remains in atomic form will strongly depend 
Table 4

Compilation of Observed Galactic [D]/[H] Ratios

\begin{tabular}{|c|c|c|c|}
\hline Target & $\begin{array}{c}d \\
(\mathrm{pc})\end{array}$ & $\log N(\mathrm{HI})$ & $\begin{array}{c}{[\mathrm{D}] /[\mathrm{H}]} \\
(\mathrm{ppm})\end{array}$ \\
\hline Sirius & 3 & 17.60 & $16.2 \pm 7.2$ \\
\hline $36 \mathrm{Oph}$ & 6 & 17.85 & $15.0 \pm 2.5$ \\
\hline$\epsilon$ Eri & 3 & 17.88 & $14.3 \pm 1.6$ \\
\hline $31 \mathrm{Com}$ & 94 & 17.88 & $20.2 \pm 1.9$ \\
\hline $\mathrm{Hz} 43$ & 68 & 17.93 & $16.6 \pm 1.4$ \\
\hline$\epsilon$ Ind & 4 & 18.00 & $16.0 \pm 2.0$ \\
\hline Procyon & 4 & 18.06 & $16.0 \pm 2.0$ \\
\hline$\beta$ Cas & 17 & 18.13 & $16.9 \pm 1.6$ \\
\hline HR 1099 & 29 & 18.13 & $14.6 \pm 1.0$ \\
\hline G191-B2B & 69 & 18.18 & $16.6 \pm 4.1$ \\
\hline$\beta \mathrm{CMa}$ & 153 & 18.20 & $\geqslant 16$ \\
\hline$\sigma$ Gem & 37 & 18.20 & $13.8 \pm 2.1$ \\
\hline Capella & 13 & 18.24 & $15.9 \pm 1.5$ \\
\hline$\beta$ Gem & 10 & 18.26 & $14.8 \pm 2.2$ \\
\hline$\alpha$ Tri & 20 & 18.33 & $13.3 \pm 2.0$ \\
\hline$\beta$ Cet & 29 & 18.36 & $21.9 \pm 6.8$ \\
\hline$\lambda$ And & 26 & 18.45 & $17.0 \pm 5.0$ \\
\hline Feige 24 & 74 & 18.47 & $13.0 \pm 5.0$ \\
\hline WD 0621-376 & 78 & 18.70 & $14.1 \pm 6.0$ \\
\hline WD 2211-495 & 53 & 18.76 & $15.1 \pm 6.5$ \\
\hline WD 1634-573 & 37 & 18.85 & $15.8 \pm 2.5$ \\
\hline$\alpha \mathrm{Vir}$ & 80 & 19.00 & $15.8 \pm 8.0$ \\
\hline GD 246 & 79 & 19.11 & $15.1 \pm 1.9$ \\
\hline$\lambda \operatorname{Sco}$ & 216 & 19.23 & $7.6 \pm 1.8$ \\
\hline$\beta$ Cen & 161 & 19.63 & $11.7 \pm 7.5$ \\
\hline$\gamma \mathrm{Vel}$ & 258 & 19.71 & $21.8 \pm 2.1$ \\
\hline$\alpha \mathrm{Cru}$ & 98 & 19.85 & $12.6 \pm 3.6$ \\
\hline $\mathrm{BD}+284211$ & 104 & 19.85 & $13.9 \pm 1.0$ \\
\hline Lan 23 & 122 & 19.89 & $21.9 \pm 10.8$ \\
\hline$\mu \mathrm{Col}$ & 400 & 19.86 & $6.9 \pm 6.9$ \\
\hline$\zeta$ Pup & 429 & 19.96 & $14.0 \pm 2.3$ \\
\hline TD1 32709 & 520 & 20.03 & $18.6 \pm 5.3$ \\
\hline WD $1034+001$ & 155 & 20.07 & $21.4 \pm 5.3$ \\
\hline $\mathrm{BD}+393226$ & 290 & 20.08 & $11.7 \pm 3.1$ \\
\hline Feige 110 & 179 & 20.14 & $21.4 \pm 5.7$ \\
\hline$\gamma$ Cas & 188 & 20.16 & $9.8 \pm 2.7$ \\
\hline$\iota$ Ori & 407 & 20.15 & $14.1 \pm 2.8$ \\
\hline$\delta$ Ori & 281 & 20.19 & $7.4 \pm 1.2$ \\
\hline$\theta$ Car & 135 & 20.28 & $5.0 \pm 3.4$ \\
\hline$\epsilon$ Ori & 412 & 20.45 & $6.3 \pm 1.8$ \\
\hline PG $0038+199$ & 297 & 20.48 & $19.1 \pm 2.6$ \\
\hline LSE 44 & 554 & 20.52 & $22.4 \pm 9.0$ \\
\hline JL 9 & 590 & 20.78 & $10.0 \pm 1.9$ \\
\hline HD 195965 & 794 & 20.95 & $8.5 \pm 1.6$ \\
\hline LSS 1274 & 580 & 20.98 & $7.6 \pm 1.9$ \\
\hline HD 191877 & 2200 & 21.05 & $7.8 \pm 2.4$ \\
\hline HD 41161 & 1253 & 21.08 & $21.6 \pm 5.1$ \\
\hline HD 53975 & 1318 & 21.14 & $10.2 \pm 2.3$ \\
\hline HD 90087 & 2740 & 21.22 & $8.7 \pm 1.7$ \\
\hline
\end{tabular}

Notes. Data taken from Linsky et al. (2006), Oliveira \& Hébrard (2006), and Ellison et al. (2007).

on the density and temperature of the cloud. Furthermore, if the cloud is immersed in an ISRF, the photodissociation of molecules by the impinging UV photons will also affect the amount of atomic deuterium and hydrogen present in the cloud envelope. The self-shielding of $\mathrm{H}_{2}$ means that, as extinction into the cloud increases, its photodissociation rate falls off more rapidly than that of HD (for a detailed discussion, see Le Petit et al. 2002). This difference in shielding results in a significant fraction of atomic deuterium being maintained to greater cloud depth, whereas atomic hydrogen
Table 5

Compilation of Observed Galactic $[\mathrm{HD}] / 2\left[\mathrm{H}_{2}\right]$ Ratios

\begin{tabular}{|c|c|c|c|c|c|}
\hline Target & $\begin{array}{c}d \\
(\mathrm{pc})\end{array}$ & $\begin{array}{c}A_{V} \\
(\mathrm{mag})\end{array}$ & $\log N\left(\mathrm{H}_{\mathrm{tot}}\right)$ & $f\left(\mathrm{H}_{2}\right)$ & $\begin{array}{c}{[\mathrm{HD}] / 2\left[\mathrm{H}_{2}\right]} \\
(\mathrm{ppm})\end{array}$ \\
\hline HD 12323 & 3900 & 0.72 & 21.29 & 0.21 & $2.6 \pm 1.1$ \\
\hline HD 15558 & 2187 & 2.44 & 21.69 & 0.32 & $2.8 \pm 2.1$ \\
\hline HD 24534 & 398 & 2.05 & 21.34 & 0.76 & $4.6 \pm 4.2$ \\
\hline HD 27778 & 220 & 1.01 & 21.34 & 0.56 & $6.3 \pm 16.0$ \\
\hline HD 45314 & 799 & 2.03 & 21.28 & 0.42 & $2.5 \pm 2.1$ \\
\hline HD 53367 & 247 & 1.76 & 21.65 & 0.51 & $2.5 \pm 2.0$ \\
\hline HD 73882 & 925 & 2.36 & 21.58 & 0.67 & $2.5 \pm 6.8$ \\
\hline HD 74920 & 1497 & 1.09 & 21.25 & 0.21 & $5.9 \pm 0.5$ \\
\hline HD 90087 & 2716 & 0.93 & 21.18 & 0.08 & $1.7 \pm 0.3$ \\
\hline HD 91651 & 3500 & 1.01 & 21.16 & 0.02 & $1.1 \pm 1.2$ \\
\hline HD 91824 & 4000 & 0.87 & 21.19 & 0.09 & $1.1 \pm 0.2$ \\
\hline HD 93204 & 2630 & 1.30 & 21.43 & 0.04 & $1.4 \pm 0.3$ \\
\hline HD 93205 & 2600 & 1.20 & 21.35 & 0.05 & $1.7 \pm 0.3$ \\
\hline HD 93206 & 2512 & 1.21 & 21.35 & 0.03 & $2.3 \pm 0.5$ \\
\hline HD 93222 & 2900 & 1.71 & 21.55 & 0.03 & $1.3 \pm 0.4$ \\
\hline HD 94493 & 3327 & 0.62 & 21.17 & 0.18 & $0.7 \pm 0.5$ \\
\hline HD 101131 & 709 & 1.05 & $\ldots$ & $\ldots$ & $1.9 \pm 0.8$ \\
\hline HD 101190 & 2399 & 0.89 & 21.29 & 0.27 & $2.4 \pm 0.7$ \\
\hline HD 101413 & 2399 & 1.12 & 21.34 & 0.22 & $2.1 \pm 1.5$ \\
\hline HD 101436 & 2399 & 1.18 & 21.34 & 0.22 & $4.8 \pm 1.9$ \\
\hline HD 104705 & 3898 & 0.73 & 21.16 & 0.12 & $1.1 \pm 1.3$ \\
\hline HD 110432 & 301 & 2.02 & 21.20 & 0.55 & $3.1 \pm 0.9$ \\
\hline HD 116852 & 4760 & 0.53 & 21.01 & 0.12 & $1.2 \pm 0.4$ \\
\hline HD 147888 & 136 & 1.91 & 21.71 & 0.18 & $4.2 \pm 14.1$ \\
\hline HD 148422 & 8836 & 0.85 & 21.23 & 0.16 & $4.4 \pm 2.8$ \\
\hline HD 149404 & 1380 & 2.23 & 21.57 & 0.33 & $4.5 \pm 1.3$ \\
\hline HD 152233 & 1905 & 1.33 & 21.37 & 0.17 & $2.3 \pm 1.0$ \\
\hline HD 152248 & 1758 & 1.66 & $\ldots$ & $\ldots$ & $3.6 \pm 1.5$ \\
\hline HD 152723 & 1905 & 1.41 & 21.49 & 0.13 & $2.6 \pm 0.8$ \\
\hline HD 161807 & 383 & 0.34 & $\ldots$ & $\ldots$ & $0.9 \pm 0.1$ \\
\hline HD 177989 & 4909 & 0.71 & 21.06 & 0.23 & $3.2 \pm 1.6$ \\
\hline HD 185418 & 950 & 1.16 & 21.56 & 0.47 & $2.8 \pm 2.1$ \\
\hline HD 192639 & 1100 & 1.87 & 21.47 & 0.32 & $3.8 \pm 2.0$ \\
\hline HD 199579 & 794 & 1.09 & 21.25 & 0.38 & $1.8 \pm 0.4$ \\
\hline HD 201345 & 1907 & 0.53 & 20.91 & 0.03 & $1.9 \pm 0.5$ \\
\hline HD 206267 & 850 & 1.41 & 21.54 & 0.42 & $3.6 \pm 1.2$ \\
\hline HD 207198 & 832 & 1.50 & 21.55 & 0.38 & $9.7 \pm 10.6$ \\
\hline HD 207538 & 832 & 1.44 & 21.58 & 0.43 & $4.2 \pm 2.5$ \\
\hline HD 224151 & 1355 & 1.11 & 21.45 & 0.26 & $1.1 \pm 0.2$ \\
\hline HD 303308 & 2630 & 1.30 & 21.50 & 0.11 & $2.3 \pm 0.7$ \\
\hline HD 308813 & 2398 & 0.87 & 21.26 & 0.22 & $0.8 \pm 0.2$ \\
\hline
\end{tabular}

Notes. Data taken from Snow et al. (2008), with additional extinction and $N\left(\mathrm{H}_{2}\right)$ data taken from Rachford et al. (2002, 2009). For targets without $R_{V}$ data, a typical value of 3.1 is adopted when calculating the visual extinction $\left[A_{V}=R_{V} \cdot E(B-V)\right]$.

rapidly transitions to molecular form. Model clouds containing density and temperature gradients, or being exposed to an external radiation field, will therefore yield depth-dependent $\mathrm{D} / \mathrm{H}$ ratios.

Figure 10 shows the atomic $\mathrm{D} / \mathrm{H}$ ratio determined from the ratio of abundances, $[\mathrm{D}] /[\mathrm{H}]$, as a function of visual extinction and radius in our static cloud model (i.e., with no diffusion). It is clear that the chemical processes discussed above can lead to a dramatic variation of the $\mathrm{D} / \mathrm{H}$ ratio within such a cloud, with values spanning four orders of magnitude across its radius. With increasing depth (or visual extinction) into the cloud, the $\mathrm{D} / \mathrm{H}$ ratio is seen to rise sharply, as hydrogen rapidly transitions to $\mathrm{H}_{2}$ due to its effective shielding, while the density of atomic deuterium remains high, with a more gradual transition from $\mathrm{D}$ to $\mathrm{HD}$, owing to its poor shielding. 

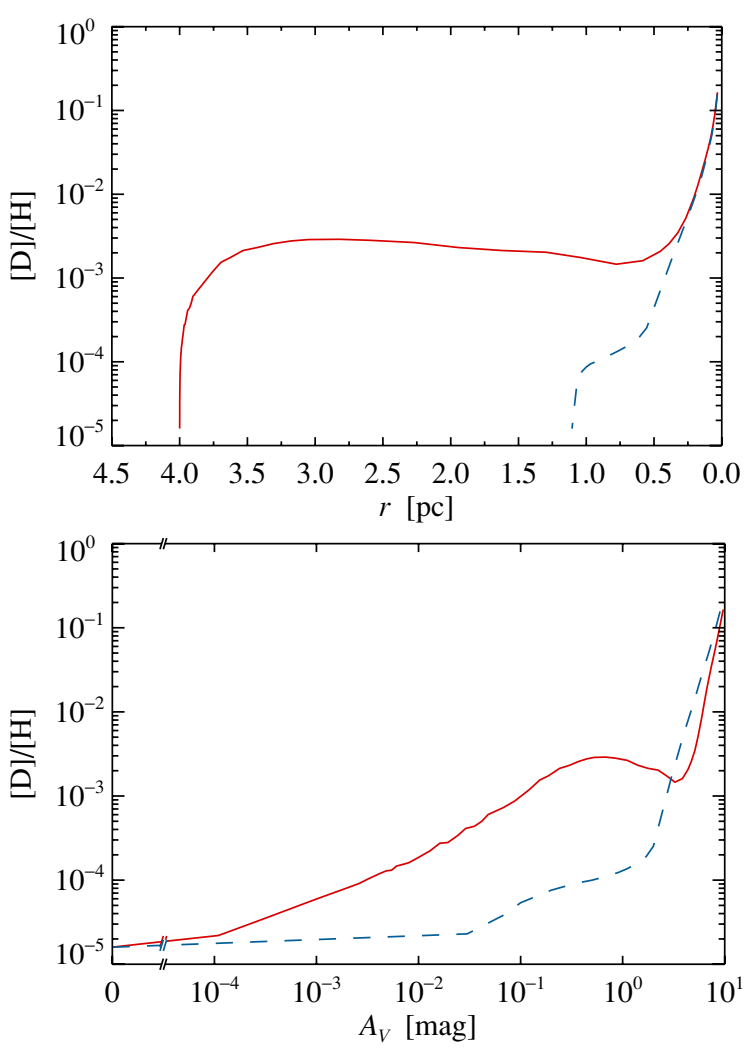

Figure 10. Atomic D/H ratio for static (i.e., no diffusion) versions of models 1 (blue, dashed) and 2 (red, solid), both at $t=1 \mathrm{Myr}$. The top plot shows the radial dependence of the $\mathrm{D} / \mathrm{H}$ ratio and the bottom plot shows its variation as a function of visual extinction into the cloud.

(A color version of this figure is available in the online journal.)

Going deeper into the cloud, the atomic $\mathrm{D} / \mathrm{H}$ ratio levels off as hydrogen becomes predominantly molecular and deuterium remains in atomic form, before the ratio begins to drop again, as deuterium, too, moves to molecular form (primarily HD). Finally, in the dark cloud interior $\left(A_{V}>1 \mathrm{mag}\right)$, the $\mathrm{D} / \mathrm{H}$ ratio starts to rise again as deuteration of $\mathrm{H}_{3}^{+}$and its subsequent dissociative recombination preferentially leads to the formation of $\mathrm{D}$, rather than $\mathrm{H}$, atoms in the cold dense core. Fractionation is so effective in the core that the atomic $\mathrm{D} / \mathrm{H}$ ratio exceeds 0.1 , a factor of $10^{4}$ increase over the cosmic $\mathrm{D} / \mathrm{H}$ ratio.

As we have seen in Section 3, introducing turbulent mixing to the model changes the amount of atomic deuterium and hydrogen present in the cloud by causing these atomic species to diffuse along their abundance gradients. Changes in the abundance of $\mathrm{H}$ and $\mathrm{D}$ due to turbulent diffusion lead to a corresponding change in the atomic $\mathrm{D} / \mathrm{H}$ ratio. To examine the effect of increasing turbulent diffusion on the $\mathrm{D} / \mathrm{H}$ ratio in the outer regions of the cloud, we focus our attention on the results of model 2, which includes a warm diffuse envelope that better represents the conditions at the edges of molecular clouds.

In Section 3.3, we showed that the abundances of atomic deuterium and hydrogen tend to drop at the cloud edge as the level of turbulence increases. This is due to their inward diffusion along the abundance gradients that are created by the $\mathrm{H} / \mathrm{H}_{2}$ and $\mathrm{D} / \mathrm{HD}$ transitions. However, the strength of this diffusion is not the same for both species, and the abundance profile of atomic hydrogen is changed more significantly than that of atomic deuterium. This leads to changes in the atomic $\mathrm{D} / \mathrm{H}$ ratio as the level of turbulent diffusion is increased, as shown in Figure 11.

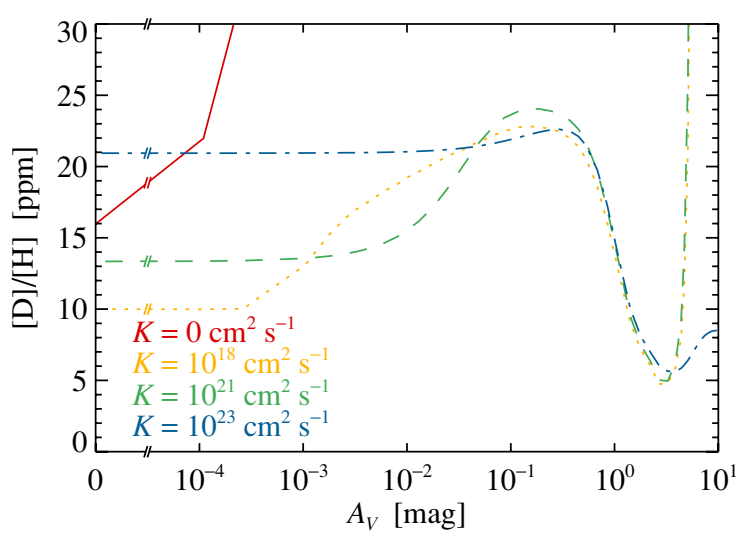

Figure 11. Atomic $\mathrm{D} / \mathrm{H}$ ratio as a function of visual extinction into the cloud for diffusion coefficients of $K=0,10^{18}, 10^{21}$, and $10^{23} \mathrm{~cm}^{2} \mathrm{~s}^{-1}$ (red solid, yellow dotted, green dashed, and blue dot-dashed lines, respectively). The results shown are for model 2 at $t=1$ Myr.

(A color version of this figure is available in the online journal.)

At $K=10^{18} \mathrm{~cm}^{2} \mathrm{~s}^{-1}$, the $\mathrm{D} / \mathrm{H}$ ratio at the cloud edge drops from the elemental value (16 ppm) to about $10 \mathrm{ppm}$. This is due to the drop in atomic deuterium abundance while the abundance of hydrogen remains relatively unchanged. As the level of turbulence increases, the inward diffusion of atomic hydrogen becomes more active and its abundance at the cloud edge begins to drop. Atomic deuterium is less affected, since its abundance gradient is smaller and occurs deeper into the cloud. The net result is that the $\mathrm{D} / \mathrm{H}$ ratio begins to rise again, reaching $21 \mathrm{ppm}$ when $K=10^{23} \mathrm{~cm}^{2} \mathrm{~s}^{-1}$, the maximum diffusion coefficient we consider here.

Deeper into the cloud, the introduction of turbulent diffusion smoothes out the abundance profiles of atomic deuterium and hydrogen, leading to less variation in the atomic $\mathrm{D} / \mathrm{H}$ profile with cloud depth. In the cloud envelope $\left(A_{V}<5\right.$ mag), even a low level of turbulent mixing can dramatically reduce the variation of the $\mathrm{D} / \mathrm{H}$ ratio, from over two orders of magnitude when no turbulent mixing is present to just a factor of $\sim 4$ when $K=10^{18} \mathrm{~cm}^{2} \mathrm{~s}^{-1}$. In the cloud core $\left(A_{V} \sim 10 \mathrm{mag}\right)$, turbulent diffusion has less effect on the atomic $\mathrm{D} / \mathrm{H}$ ratio, which only drops by a factor of 10 when $K=10^{21} \mathrm{~cm}^{2} \mathrm{~s}^{-1}$, owing to the greater influx of atomic hydrogen and the reduced abundance of deuterated $\mathrm{H}_{3}^{+}$, the main source of $\mathrm{D}$ atoms in the cold dense core. Increasing the diffusion coefficient to $K=10^{23} \mathrm{~cm}^{2} \mathrm{~s}^{-1}$, however, causes the $\mathrm{D} / \mathrm{H}$ ratio in the core to drop to $\approx 9 \mathrm{ppm}$, a factor of $10^{4}$ reduction compared to the static model with no diffusion. Strong turbulence can therefore have a bigger impact on the atomic $\mathrm{D} / \mathrm{H}$ ratio in the core. At this level of turbulence, diffusive mixing is so effective that the abundance of atomic hydrogen is fairly uniform across the cloud, varying by less than a factor of five, whereas, in the static model, it is nearly $10^{5}$ lower at the core than at the edge.

The effect of varying the strength of turbulent diffusion in the model is therefore to produce a range of atomic $\mathrm{D} / \mathrm{H}$ ratios at the cloud edge, with values spanning 10-20 ppm. In the cloud interior, increasing turbulence causes a more significant drop in the atomic $\mathrm{D} / \mathrm{H}$ ratio, by up to four orders of magnitude in the case of strong diffusion $\left(K=10^{23} \mathrm{~cm}^{2} \mathrm{~s}^{-1}\right)$.

If the lines of sight along which the galactic atomic $\mathrm{D} / \mathrm{H}$ ratio has been measured are assumed to intersect diffuse gas at the edges of molecular clouds, the variation of the observed column densities of D I and $\mathrm{HI}_{\mathrm{I}}$ (and therefore the inferred $\mathrm{D} / \mathrm{H}$ ratio) will be dependent upon the degree of 


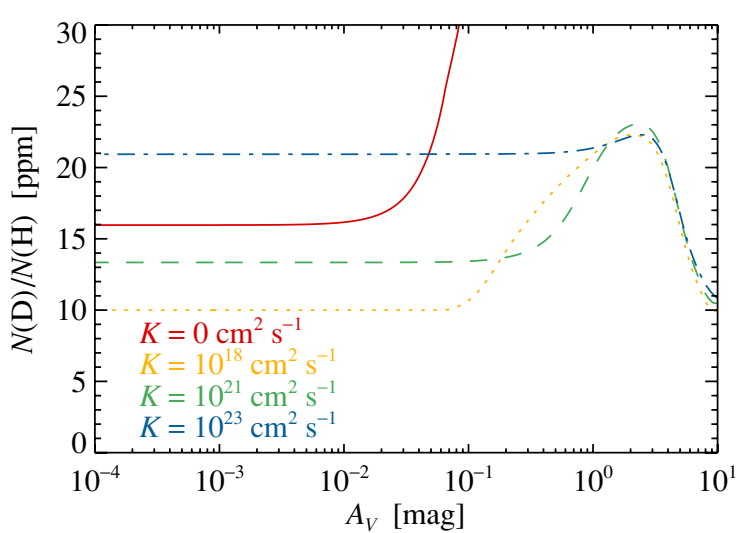

Figure 12. Integrated line-of-sight atomic D/H ratio through the model cloud, i.e., $N(\mathrm{D}) / N(\mathrm{H})$, along sightlines of increasing extinction through the cloud, for diffusion coefficients of $K=0,10^{18}, 10^{21}$, and $10^{23} \mathrm{~cm}^{2} \mathrm{~s}^{-1}$ (red solid, yellow dotted, green dashed, and blue dot-dashed lines, respectively). The results shown are for model 2 at $t=1 \mathrm{Myr}$.

(A color version of this figure is available in the online journal.)

turbulent mixing within the clouds. Figure 12 shows the atomic $\mathrm{D} / \mathrm{H}$ ratio that would be observed along a line of sight through a spherically symmetric model cloud, as a function of visual extinction through the cloud, for the case of no turbulent diffusion $\left(K=0 \mathrm{~cm}^{2} \mathrm{~s}^{-1}\right)$ up to strong diffusion $\left(K=10^{23} \mathrm{~cm}^{2} \mathrm{~s}^{-1}\right)$. It can be seen that, even for sightlines that intersect the cloud near its edge, the atomic $\mathrm{D} / \mathrm{H}$ ratio can change by over a factor of two. If clouds exhibiting varying degrees of turbulent mixing are assumed to exist along different sightlines (as is almost certainly the case), this could help to account for the observed range of atomic $\mathrm{D} / \mathrm{H}$ ratios.

Other mechanisms invoked to explain the observed variation in the atomic $\mathrm{D} / \mathrm{H}$ ratio (in particular, depletion of deuterium onto dust grains or galactic infall of nearly pristine material) are expected to also produce variations in the observed metal abundances. In the case of the deuterium depletion model, the observed abundances of refractory metals are predicted to vary in line with that of deuterium, since they exhibit similar depletion onto grains. Conversely, increases in the $\mathrm{D} / \mathrm{H}$ ratio produced by unmixed infalling low-metallicity material should be accompanied by decreased metal abundances. However, our turbulent diffusion models show only minor variations $(<5 \%)$ in the metal abundances (O I, Fe II, and Mg II) that would be observed at the cloud surface over the full range of turbulent diffusion coefficients considered. This suggests that there would be only a weak correlation between the $\mathrm{D} / \mathrm{H}$ ratio and the metal abundances if turbulent diffusion were the sole cause of variation in the atomic $\mathrm{D} / \mathrm{H}$ ratio.

Figure 13 shows the molecular $\mathrm{D} / \mathrm{H}$ ratio determined from the ratio of abundances, $[\mathrm{HD}] / 2\left[\mathrm{H}_{2}\right]$, as a function of visual extinction into the cloud. In comparison to the atomic $\mathrm{D} / \mathrm{H}$ ratio, the molecular equivalent shows significantly less variation across the cloud (two orders of magnitude, instead of four orders of magnitude). In the static cloud model, the molecular $\mathrm{D} / \mathrm{H}$ ratio attains the elemental $\mathrm{D} / \mathrm{H}$ ratio $(16 \mathrm{ppm})$ at the cloud edge, since the same unattenuated photodissociation rate is assumed for $\mathrm{H}_{2}$ and $\mathrm{HD}$, before dropping rapidly to $<1 \mathrm{ppm}$ when $A_{V} \gtrsim 10^{-4} \mathrm{mag}$, as self-shielding by $\mathrm{H}_{2}$ causes its abundance to rise sharply. Deeper into the cloud $\left(A_{V} \sim 0.1 \mathrm{mag}\right)$, the abundance of HD begins to rise, as the density increases and its rate of photodissociation starts to drop off, while the abundance of $\mathrm{H}_{2}$ remains fairly constant. This leads to a steady increase in the $[\mathrm{HD}] / 2\left[\mathrm{H}_{2}\right]$ ratio, which peaks at about $12 \mathrm{ppm}$. In the

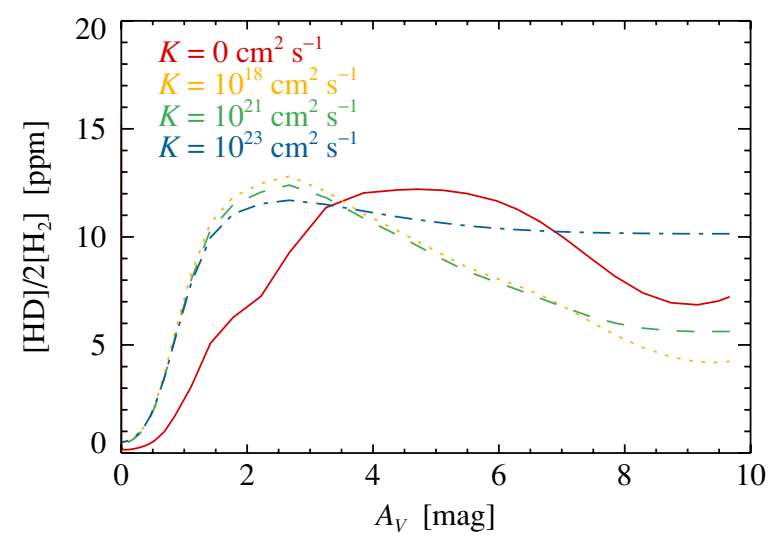

Figure 13. Molecular $[\mathrm{HD}] / 2\left[\mathrm{H}_{2}\right]$ ratio as a function of visual extinction into the cloud for diffusion coefficients of $K=0,10^{18}, 10^{21}$, and $10^{23} \mathrm{~cm}^{2} \mathrm{~s}^{-1}$ (red solid, yellow dotted, green dashed, and blue dot-dashed lines, respectively). The results shown are for model 2 at $t=1 \mathrm{Myr}$.

(A color version of this figure is available in the online journal.)

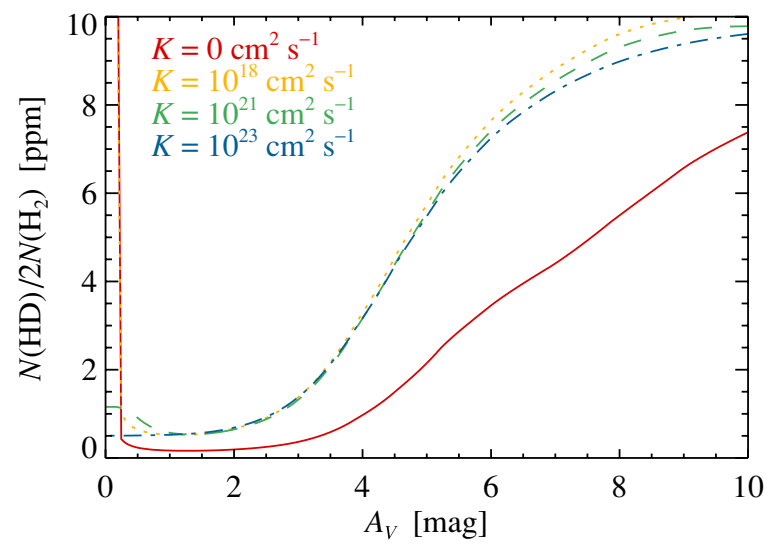

Figure 14. Integrated line-of-sight molecular $\mathrm{D} / \mathrm{H}$ ratio through the model cloud, i.e., $N(\mathrm{HD}) / 2 N\left(\mathrm{H}_{2}\right)$, along sightlines of increasing extinction through the cloud, for diffusion coefficients of $K=0,10^{18}, 10^{21}$, and $10^{23} \mathrm{~cm}^{2} \mathrm{~s}^{-1}$ (red solid, yellow dotted, green dashed, and blue dot-dashed lines, respectively). The results shown are for model 2 at $t=1 \mathrm{Myr}$.

(A color version of this figure is available in the online journal.)

cloud core, efficient fractionation transfers the deuterium from HD to other molecules (and to atomic D), and the molecular $\mathrm{D} / \mathrm{H}$ ratio begins to fall again, reaching $7 \mathrm{ppm}$ at the center.

The influence of turbulent diffusion on the molecular $\mathrm{D} / \mathrm{H}$ ratio serves to lower the ratio at the cloud edge (to $\sim 1 \mathrm{ppm}$ when $K=10^{21} \mathrm{~cm}^{2} \mathrm{~s}^{-1}$ ), since the mixing is more effective at bringing $\mathrm{H}_{2}$ to the cloud surface than HD. The outward shift of the D/HD transition caused by turbulent diffusion (see Section 3.3) means that [HD]/2[H $\left[\mathrm{H}_{2}\right]$ begins to rise at lower $A_{V}$, and the ratio peaks at 2-3 mag, compared to 4-5 mag in the static cloud model. This shift is insensitive to the strength of turbulent diffusion. Changes in the level of fractionation brought about by turbulent mixing affect the amount of deuterium transferred from $\mathrm{HD}$ to other molecules and lead to molecular $\mathrm{D} / \mathrm{H}$ ratios varying between 5 and $10 \mathrm{ppm}$ at the cloud center.

Molecular D/H ratios determined from line-of-sight column densities through the model cloud, $N(\mathrm{HD}) / 2 N\left(\mathrm{H}_{2}\right)$, are shown as a function of line-of-sight visual extinction in Figure 14 for turbulent diffusion coefficients of 0 to $10^{23} \mathrm{~cm}^{2} \mathrm{~s}^{-1}$. Comparing this figure to the observed molecular D/H ratios in Figure 9, which have been measured for sightlines showing up to $2.5 \mathrm{mag}$ of extinction, it is clear that our models underpredict the ratio 
by a factor of 3-4. However, models including some degree of turbulent mixing give the best agreement, with generally higher molecular $\mathrm{D} / \mathrm{H}$ ratios over the observed range of $A_{V}$. It has been noted previously (Le Petit et al. 2002; Lacour et al. 2005) that models fail to match the observed values unless the bulk of the absorbing gas is assumed to be in a single, dense component. Since our model cloud possesses a fairly large envelope of diffuse and warm material, which dominates the line-of-sight column densities at low $A_{V}$, this could account for the difference. If the $[\mathrm{HD}] / 2\left[\mathrm{H}_{2}\right]$ abundance ratios in Figure 13 are instead compared to the observations, somewhat better agreement is found at low visual extinction ( $<1 \mathrm{mag}$ ). The agreement worsens at higher extinction, though this may be due in part to the larger uncertainties on the observed values at higher $A_{V}$.

\section{SUMMARY}

We find that turbulent diffusion affects the cloud chemistry in the following ways.

1. The ionization fraction is enhanced in the cloud center, due to an influx of ions and electrons produced by photoionization at the surface.

2. Freeze-out of abundant gas-phase species, such as $\mathrm{CO}$, is reduced, as grain mantles are photodesorbed and/or evaporated when they are brought to the cloud surface.

3. Abundances of $\mathrm{H}_{2} \mathrm{D}^{+}, \mathrm{D}_{2} \mathrm{H}^{+}$, and $\mathrm{D}_{3}^{+}$are reduced in the cloud center due to the higher ionization fraction and $\mathrm{CO}$ abundance.

4. The $\mathrm{H} / \mathrm{H}_{2}$ transition occurs significantly deeper into the cloud due to rapid inward transport of atomic hydrogen along the sharp abundance gradient caused by self-shielding of molecular hydrogen.

5. The D/HD transition is affected less by turbulent mixing, as the abundance gradients of $\mathrm{D}$ and $\mathrm{HD}$ are more gradual than those of $\mathrm{H}$ and $\mathrm{H}_{2}$ (due to the lower elemental deuterium abundance and less effective shielding of HD).

These effects lead to generally lower abundances of deuterated molecules and to a corresponding drop in fractionation ratios. We find that turbulent diffusion causes atomic hydrogen to remain abundant deep into molecular clouds, while inhibiting the pathways that normally allow significant deuteration in the cold dense cores of these clouds. This is reflected in the atomic $\mathrm{D} / \mathrm{H}$ ratio, which drops dramatically from $\sim 10^{-1}$ in the cloud center when $K=0 \mathrm{~cm}^{2} \mathrm{~s}^{-1}$ (i.e., no diffusion) to $\sim 10^{-5}$ when $K=10^{23} \mathrm{~cm}^{2} \mathrm{~s}^{-1}$. Close to the cloud edge, the atomic D/H ratio varies by over a factor of two as the level of turbulent mixing increases, with values spanning $\sim 10-20 \mathrm{ppm}$, in agreement with the range of observed values. The impact of turbulent mixing on the molecular $\mathrm{D} / \mathrm{H}$ ratio, $[\mathrm{HD}] / 2\left[\mathrm{H}_{2}\right]$, is less significant, but nevertheless shows some dependence on the degree of turbulent mixing.

Based on these results, we propose a new process to help explain the significant scatter in the observed galactic atomic $\mathrm{D} / \mathrm{H}$ ratio. If part of the intervening gas resides in the envelope of a molecular cloud, we expect the column densities of $\mathrm{H}$ I and D I near the cloud edge to be dependent on the degree of turbulent mixing within the cloud, leading to variations in the $\mathrm{D} / \mathrm{H}$ ratio that would be observed.

In reality, various mechanisms are likely to be working in combination to produce the observed range of $\mathrm{D} / \mathrm{H}$ ratios. Many of the parameters that govern the relative contribution of each process, such as the initial abundances, dust composition, turbulent diffusion coefficient, and ionization fraction, are hard to determine with any precision, making it difficult to disentangle the various processes involved, or to say definitively if one process is dominating. The turbulent diffusion mechanism proposed here could be tested by searching for a correlation between observed $\mathrm{D} / \mathrm{H}$ ratios and a proxy for the degree of turbulence along each line of sight, such as the turbulent linewidths of the absorption lines, although the analysis would be complicated by additional uncertainties due to overlapping absorption components and bulk cloud motions.

With the exception of galactic infall of low-metallicity material, all mechanisms that have so far been proposed to explain the variation in atomic $\mathrm{D} / \mathrm{H}$ ratio result in lower values. Therefore, the appropriate value to adopt would be the largest observed, i.e., 23 ppm in the Milky Way (e.g., Linsky et al. 2006). The turbulent mixing of atomic and molecular gas at the edges of molecular clouds discussed here is an alternative mechanism that can explain the scatter in atomic $\mathrm{D} / \mathrm{H}$ ratios observed beyond the Local Bubble. However, in this case the observed $\mathrm{D} / \mathrm{H}$ ratio can be either decreased or increased compared to the "true" elemental ratio, depending on the magnitude of the turbulent diffusion coefficient.

We thank W. D. Langer for helpful discussions and the referee for constructive comments which helped to improve an earlier draft of this paper. This research has been supported by the National Science Foundation grant AST-0838261 to the Caltech Submillimeter Observatory. Part of this research was carried out at the Jet Propulsion Laboratory, California Institute of Technology, under a contract with the National Aeronautics and Space Administration.

\section{REFERENCES}

Aikawa, Y., Herbst, E., Roberts, H., \& Caselli, P. 2005, ApJ, 620, 330

Bacmann, A., Lefloch, B., Ceccarelli, C., Castets, A., Steinacker, J., \& Loinard, L. 2002, A\&A, 389, L6

Bacmann, A., Lefloch, B., Ceccarelli, C., Steinacker, J., Castets, A., \& Loinard, L. 2003, ApJ, 585, L55

Barsuhn, J. 1977, A\&AS, 28, 453

Bell, T. A., Roueff, E., Viti, S., \& Williams, D. A. 2006, MNRAS, 371, 1865

Bergin, E. A., Alves, J., Huard, T., \& Lada, C. J. 2002, ApJ, 570, L101

Boland, W., \& de Jong, T. 1982, ApJ, 261, 110

Brown, P. D., \& Millar, T. J. 1989, MNRAS, 237, 661

Caselli, P., Walmsley, C. M., Tafalla, M., Dore, L., \& Myers, P. C. 1999, ApJ, 523, L165

Ceccarelli, C., Castets, A., Loinard, L., Caux, E., \& Tielens, A. G. G. M. 1998, A\&A, 338, L43

Cyburt, R. H., Fields, B. D., \& Olive, K. A. 2003, Phys. Lett. B, 567, 227

Draine, B. T. 1978, ApJS, 36, 595

Draine, B. T. 2004, in Carnegie Observatories Astrophysics Ser. 4, Origin and Evolution of the Elements, ed. A. McWilliam \& M. Rauch (Cambridge: Cambridge Univ. Press), 320

Draine, B. T. 2006, in ASP Conf. Ser. 348, Astrophysics in the Far Ultraviolet: Five Years of Discovery with FUSE, ed. G. Sonneborn, H. W. Moos, \& B.-G. Andersson (San Francisco, CA: ASP), 58

Ellison, S. L., Prochaska, J. X., \& Lopez, S. 2007, MNRAS, 380, 1245

Federman, S. R., \& Allen, M. 1991, ApJ, 375, 157

Flower, D. R., Pineau des Forêts, G., \& Walmsley, C. M. 2004, A\&A, 427, 887 Flower, D. R., Pineau des Forêts, G., \& Walmsley, C. M. 2006a, A\&A, 449, 621 Flower, D. R., Pineau des Forêts, G., \& Walmsley, C. M. 2006b, A\&A, 456, 215 Gerin, M., Pearson, J. C., Roueff, E., Falgarone, E., \& Phillips, T. G. 2001, ApJ, 551, L193

Hatchell, J. 2003, A\&A, 403, L25

Hinze, J. O. 1975, Turbulence (2nd ed.; New York: McGraw-Hill)

Hirota, T., Ikeda, M., \& Yamamoto, S. 2003, ApJ, 594, 859

Indriolo, N., Geballe, T. R., Oka, T., \& McCall, B. J. 2007, ApJ, 671, 1736

Jefferts, K. B., Penzias, A. A., \& Wilson, R. W. 1973, ApJ, 179, L57

Kramer, C., Alves, J., Lada, C. J., Lada, E. A., Sievers, A., Ungerechts, H., \& Walmsley, C. M. 1999, A\&A, 342, 257

Lacour, S., et al. 2005, A\&A, 430, 967 
Lee, H.-H., Herbst, E., Pineau des Forêts, G., Roueff, E., \& Le Bourlot, J. 1996 A\&A, 311, 690

Le Petit, F., Roueff, E., \& Le Bourlot, J. 2002, A\&A, 390, 369

Lesaffre, P., Gerin, M., \& Hennebelle, P. 2007, A\&A, 469, 949

Le Teuff, Y. H., Millar, T. J., \& Markwick, A. J. 2000, A\&AS, 146, 157

Linsky, J. L., et al. 2006, ApJ, 647, 1106

Lis, D. C., Roueff, E., Gerin, M., Phillips, T. G., Coudert, L. H., van der Tak, F. F. S., \& Schilke, P. 2002, ApJ, 571, L55

Loinard, L., Castets, A., Ceccarelli, C., Caux, E., \& Tielens, A. G. G. M. 2001, ApJ, 552, L163

Loinard, L., et al. 2002, Planet. Space Sci., 50, 1205

McCall, B. J., et al. 2003, Nature, 422, 500

Oliveira, C. M., \& Hébrard, G. 2006, ApJ, 653, 345

Parise, B., Castets, A., Herbst, E., Caux, E., Ceccarelli, C., Mukhopadhyay, I., \& Tielens, A. G. G. M. 2004, A\&A, 416, 159

Parise, B., et al. 2002, A\&A, 393, L49

Parise, B., et al. 2005, A\&A, 431, 547

Phillips, T. G., \& Huggins, P. J. 1981, ApJ, 251, 533

Phillips, T. G., \& Lis, D. C. 2006, in ASP Conf. Ser. 356, Revealing the Molecular Universe: One Antenna is Never Enough, ed. D. C. Backer, J. M. Moran, \& J. L. Turner (San Francisco, CA: ASP), 223

Phillips, T. G., \& Vastel, C. 2003, in Chemistry as a Diagnostic of Star Formation, ed. C. L. Curry \& M. Fich (Ottawa: NRC Press), 3

Prandtl, L. 1925, Z. Angew. Math. Mech., 5, 136

Prochaska, J. X., Tripp, T. M., \& Howk, J. C. 2005, ApJ, 620, L39

Prodanović, T., \& Fields, B. D. 2008, J. Cosmol. Astropart. Phys., JCAP09(2008)003

Rachford, B. L., et al. 2002, ApJ, 577, 221

Rachford, B. L., et al. 2009, ApJS, 180, 125

Roberts, H., Fuller, G. A., Millar, T. J., Hatchell, J., \& Buckle, J. V. 2002, A\&A, 381,1026

Roberts, H., Herbst, E., \& Millar, T. J. 2003, ApJ, 591, L41

Roberts, H., Herbst, E., \& Millar, T. J. 2004, A\&A, 424, 905

Roberts, H., \& Millar, T. J. 2000a, A\&A, 361, 388

Roberts, H., \& Millar, T. J. 2000b, A\&A, 364, 780
Roberts, H., \& Millar, T. J. 2007, A\&A, 471, 849

Rodgers, S. D., \& Charnley, S. B. 2001, ApJ, 553, 613

Roueff, E., Herbst, E., Lis, D. C., \& Phillips, T. G. 2007, ApJ, 661, L159

Roueff, E., Lis, D. C., van der Tak, F. F. S., Gerin, M., \& Goldsmith, P. F. 2005, A\&A, 438, 585

Roueff, E., Tiné, S., Coudert, L. H., Pineau des Forêts, G., Falgarone, E., \& Gerin, M. 2000, A\&A, 354, L63

Snow, T. P., Ross, T. L., Destree, J. D., Drosback, M. M., Jensen, A. G., Rachford, B. L., Sonnentrucker, P., \& Ferlet, R. 2008, ApJ, 688, 1124

Solomon, P. M., \& Woolf, N. J. 1973, ApJ, 180, L89

Steigman, G., Romano, D., \& Tosi, M. 2007, MNRAS, 378, 576

Tafalla, M., Myers, P. C., Caselli, P., \& Walmsley, C. M. 2004, A\&A, 416, 191

Taylor, G. I. 1915, Phil. Trans. R. Soc. A, 215, 1

Tielens, A. G. G. M. 1983, A\&A, 119, 177

Tiné, S., Roueff, E., Falgarone, E., Gerin, M., \& Pineau des Forêts, G. 2000, A\&A, 356, 1039

Tosi, M. 1996, in ASP Conf. Ser. 98, From Stars to Galaxies: the Impact of Stellar Physics on Galaxy Evolution, ed. C. Leitherer, U. Fritze-von-Alvensleben, \& J. Huchra (San Francisco, CA: ASP), 299

Turner, B. E. 2001, ApJS, 136, 579

van der Tak, F. F. S., Schilke, P., Müller, H. S. P., Lis, D. C., Phillips, T. G., Gerin, M., \& Roueff, E. 2002, A\&A, 388, L53

Vastel, C., Phillips, T. G., Ceccarelli, C., \& Pearson, J. 2003, ApJ, 593, L97

Vastel, C., Phillips, T. G., \& Yoshida, H. 2004, ApJ, 606, L127

Vasyunin, A. I., Semenov, D. A., Wiebe, D. S., \& Henning, T. 2009, ApJ, 691, 1459

Watson, W. D. 1976, Rev. Mod. Phys., 48, 513

Willacy, K. 2007, ApJ, 660, 441

Willacy, K., Langer, W. D., \& Allen, M. 2002, ApJ, 573, L119

Willacy, K., Langer, W. D., \& Velusamy, T. 1998, ApJ, 507, L171

Williams, J. P., Bergin, E. A., Caselli, P., Myers, P. C., \& Plume, R. 1998, ApJ, 503, 689

Wood, B. E., Linsky, J. L., Hébrard, G., Williger, G. M., Moos, H. W., \& Blair, W. P. 2004, ApJ, 609, 838

Xie, T., Allen, M., \& Langer, W. D. 1995, ApJ, 440, 674 